\title{
The Review Unmanned Surface Vehicle Path Planning: Based on Multi-modality Constraint
}

Chunhui Zhou ${ }^{\mathrm{a}, \mathrm{b}, \mathrm{c}}$, Shangding Gu ${ }^{\mathrm{a}, \mathrm{b} *}$, Yuanqiao Wen ${ }^{\mathrm{c}, \mathrm{d} *}$, Zhe Du ${ }^{\mathrm{e}}$, Changshi Xiao $^{\mathrm{a}, \mathrm{b}, \mathrm{c}}$, Liang Huang ${ }^{\mathrm{b}, \mathrm{c}, \mathrm{d}}$, Man Zhu ${ }^{\mathrm{b}, \mathrm{c}, \mathrm{d}}$

(a. School of Navigation, Wuhan University of Technology, Wuhan, 430063, China.

b. Hubei Key Laboratory of Inland Shipping Technology, Wuhan, 430063, China.

c. National Engineering Research Center for Water Transport Safety, Wuhan, 430063, China.

d. Intelligent Transport Systems Center, Wuhan University of Technology, Wuhan, 430063, China.

e. Department of Marine and Transport Technology, Faculty of Mechanical, Maritime and Materials Engineering, Delft University of Technology, Delft, 2628BX, The Netherlands.)

\begin{abstract}
The essence of the path planning problems is multi-modality constraint. However, most of the current literature has not mentioned this issue. This paper introduces the research progress of path planning based on the multi-modality constraint. The path planning of multi-modality constraint research can be classified into three stages in terms of its basic ingredients (such as shape, kinematics and dynamics et al.): Route Planning, Trajectory Planning and Motion Planning. It then reviews the research methods and classical algorithms, especially those applied to the Unmanned Surface Vehicle (USV) in every stage. Finally, the paper points out some existing problems in every stage and suggestions for future research.
\end{abstract}

\section{KEYWORDS}

Unmanned Surface Vehicle (USV); $\quad$ Path Planning; $\quad$ Route Planning; Trajectory Planning; Motion Planning; Multi-modality Constraint

*Corresponding Author. E-mail address: gshangd@163.com

${ }^{*}$ Corresponding Author. E-mail address: 3444324@qq.com 


\section{INTRODUCTION.}

\subsection{BACKGROUND INTRODUCTION}

With the development and application of artificial intelligence and machine learning, more and more studies focus on unmanned vehicles and their applications (Zhou, Z., 2016). For example, Unmanned Ground Vehicle (UGV) or wheeled robot is widely used in field of industrial automation (automatic forklift), warehouse management, planet exploring (lunar rover), disaster rescue, intelligent transportation (automatic drive) and military operation (de-mining robot) (Arai et al., 2002; Farinelli et al., 2004; Kui et al., 2007). The application of Unmanned Aerial Vehicle (UAV) is also increasingly changed from military domain to civil use, such as remote sensing photographing, agricultural spraying, communications relay, environmental monitoring and express service (Jayoung et al., 2013; George et al., 2012; Mingzhu et al., 2016). The development of UGV and UAV has already been updated to a new level.

Another unmanned vehicle should also be paid attention to, which is the Unmanned Surface Vehicle (USV). The application scenarios are not widely applied for civil use and the studies of a USV are relatively fewer and commence a bit late. However, USVs have played an important role in some areas like ocean sampling, seabed mapping, network monitoring cooperating with UAV and UGV, search and rescue, and harbor patrolling (Shafer, et al., 2008; Simetti, et al., 2009; Steimle, E. T. \& Hall, M. L., 2006; Vitor A. M. Jorge et al., 2019; Liu, Z. et al., 2016). In addition, USVs also have great potential in urban waterways cargo transport for relieving the congestion in the overloaded road networks (Chen, L., et al., 2019). Nowadays, many countries become more and more interested in rich maritime resources and some of them have already cataloged this into their national development strategy. The USV is an important tool for exploring oceans (Fossen, T.I. 1994; Liu, Z. et al., 2016). Thus it should be well studied.

No matter what type the unmanned vehicles are, a vital character for all of them is autonomy. The word "autonomy" is originated from the ancient Greece, which consists

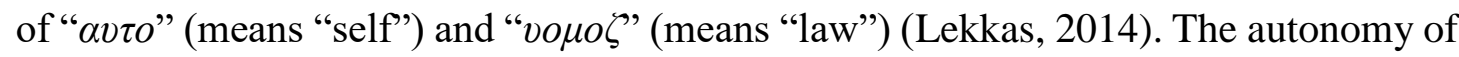
unmanned vehicles is the ability that can make a plan according to some rules (laws) and execute it all by themselves in a certain environment in the absence of human intervention. Especially, a very important part of autonomy is to work out a safe path for the vehicle, also called Path Planning (Murphy, R. 2000; H. Choset, 2005). Path planning is one of the key technologies in the process of automating and intelligentizing USVs and carrying out complex tasks of USVs (Fossen, T.I. 2002; Lazarowska A., 
2015; Song, R. et al., 2017).

When carrying out researches on Path Planning, many researchers always cite Durrant-Whyte Hugh's three questions to describe the issue, "Where am I?", "Where am I going?" and "How do I get there?" (Durrant-Whyte Hugh, 1994). These questions are applicable to an early stage of Path Planning, because at this stage both the planning environment (like indoor or virtual environment) and the research objects (like game character or wheeled robot) were relatively simple. When it comes to a UGV and a UAV, due to the circumstances either fixed (the urban road network) or ample (the expansive airspace), and relatively easy control (they can be controlled positioning, like braking and hovering), the three questions are appropriate.

For the USV, the environment is far more complex. Apart from the interference of wind, wave and current, the USV's inertia, in some environments the resistance and the response time on waters are also larger than those on the ground and in the air (Fossen, T.I. et al., 2017; Zhang, F. et al., 2015). The control of the USV is harder than the UGV and the UAV to some extent. Thus, the method on traditional path planning and these three questions might be not applicable to the USV. In fact, the path planning of the USV requires more description, such as the USV's situation, response time and inertia factor, etc. And the "old" three questions should be changed to new four questions, "What's my situation?", "Where can I go?", "Where should I go?" and "How should I do to get there" (Table 1 shows the contrast and analysis of UGVs, UAVs and USVs, Table 2 shows the USVs research For various applications). In addition, we referenced the literature (Jin K. F., et al., 2018) that illustrated the 5 levels of USVs automation and intelligent. Further, we expanded and refined the USVs automation and intelligent level classification (Table 3 shows the USVs automation and intelligent level classification).

Table 1. UGVs, UAVs and USVs Contrast and Analysis

\begin{tabular}{cccc}
\hline Type & UAVs & UGVs & USVs \\
\hline $\begin{array}{c}\text { Motion speed \& } \\
\text { inertia } \\
\text { Major disturbing } \\
\text { factors }\end{array}$ & $\begin{array}{c}\text { Fast motion and } \\
\text { small inertia }\end{array}$ & $\begin{array}{c}\text { Fast motion and } \\
\text { small inertia }\end{array}$ & $\begin{array}{c}\text { Fast motion and } \\
\text { great inertia } \\
\text { Wind, current and }\end{array}$ \\
$\begin{array}{c}\text { Wunning scenarios } \\
\text { The expansive } \\
\text { airspace }\end{array}$ & The road network & $\begin{array}{c}\text { The waters } \\
\text { environment }\end{array}$ \\
Response time & Small & Small & Large \\
Application & Remote sensing & Industrial & Ocean sampling, \\
scenarios & photographing, & automation, & seabed mapping, \\
\hline
\end{tabular}




\begin{tabular}{ccc}
\hline $\begin{array}{c}\text { agricultural } \\
\text { spraying, } \\
\text { communications } \\
\text { relay, }\end{array}$ & $\begin{array}{c}\text { warehouse } \\
\text { management, } \\
\text { planet exploring, } \\
\text { disaster rescue, } \\
\text { environmental } \\
\text { monitoring and } \\
\text { express service, }\end{array}$ & $\begin{array}{c}\text { network monitoring } \\
\text { cooperating with } \\
\text { UAVs and }\end{array}$ \\
etc. & $\begin{array}{c}\text { transportation and } \\
\text { military operation, }\end{array}$ & $\begin{array}{c}\text { and rescue, and } \\
\text { harbor patrolling, }\end{array}$ \\
& etc. & etc. \\
\hline
\end{tabular}

Table 2 USVs of Various Applications

\begin{tabular}{|c|c|}
\hline $\begin{array}{l}\text { USV use } \\
\text { division }\end{array}$ & Specific applications type \\
\hline $\begin{array}{c}\text { Water } \\
\text { sampling and } \\
\text { monitoring }\end{array}$ & $\begin{array}{l}\text { USV for water quality monitoring and measurement (T. H. } \\
\text { Yang et al., 2018), USV for shore monitoring (G. Hitz et al., } \\
\text { 2016), USVs as environmental monitoring and assessment } \\
\text { tools (E.T., 2006; W. Naeem et al., 2008), Key technology of } \\
\text { water sample collection for USV (F. Li, 2016). }\end{array}$ \\
\hline Antisubmarine & $\begin{array}{l}\text { USV anti-submarine and combat mode (W.Z. Li et al., 2018), } \\
\text { USV antisubmarine and load analysis (J. T. Su, 2018), } \\
\text { Unmanned vehicles for anti-submarine warfare (Fahey, S., \& } \\
\text { Luqi. 2016). }\end{array}$ \\
\hline $\begin{array}{c}\text { Target } \\
\text { tracking }\end{array}$ & $\begin{array}{l}\text { Time-space fusion of USV target tracking (Y. Peng et al., } \\
\text { 2018), USV target detection and tracking based on ssd-cf (X. } \\
\text { J. Chen et al., 2019), Target path tracking control } \\
\text { optimization for USV (X. Y. Cong et al., 2019), Target } \\
\text { detection and tracking of USV based on optical vision (W. J. } \\
\text { Zeng, 2013). }\end{array}$ \\
\hline $\begin{array}{l}\text { Maritime } \\
\text { search and } \\
\text { rescue }\end{array}$ & $\begin{array}{l}\text { USV water search and rescue (A. J. Shafer et al., 2008; G. A. } \\
\text { Wilde and R. R. Murphy 2018), Power design for search and } \\
\text { rescue USV (J. Wang et al., 2017), The direction and speed } \\
\text { control of search and rescue USV (X. D. Tan, 2019). }\end{array}$ \\
\hline Patrol USV & $\begin{array}{l}\text { Patrol USV based on metacentric automatic adjustment } \\
\text { function (S. Zhao et al., 2018), USV information collection } \\
\text { and patrol (G. Oriolo et al., 1995), USVs operating in harbour } \\
\text { fields (Casalino, G. et al., 2009); inspect littoral structures } \\
\text { (Steimle, E., et al., 2009); complete coverage path planning } \\
\text { of USV for island mapping (Yuxuan, Z., et al., 2017) }\end{array}$ \\
\hline
\end{tabular}


Table 3 USVs automation and intelligent level classification

\begin{tabular}{|c|c|c|}
\hline Classification & Level Description & Current Research Status \\
\hline Level one & $\begin{array}{l}\text { Remotely control and } \\
\text { plan the ship's path. }\end{array}$ & $\begin{array}{l}\text { have achieved (Caccia, } \\
\text { M. et al., 2008) }\end{array}$ \\
\hline Level two & $\begin{array}{l}\text { The ability of path } \\
\text { tracking. }\end{array}$ & $\begin{array}{c}\text { have achieved } \\
\text { (W. J. Zeng, 2013; X. J. } \\
\text { Chen et al., 2019) } \\
\text { have achieved }\end{array}$ \\
\hline Level three & $\begin{array}{l}\text { Optimal path planning } \\
\text { capability under static } \\
\text { obstacles. }\end{array}$ & $\begin{array}{l}\text { (Thakur, A. er al., 2011; } \\
\text { Blaich, M. et al., 2012; } \\
\text { Song, R. et al., 2019; } \\
\text { Xiaojie Sun, 2016; Lei X. } \\
\quad \text { et al., 2019;) }\end{array}$ \\
\hline Level four & $\begin{array}{l}\text { Under dynamic obstacles, } \\
\text { better path planning is } \\
\text { achieved. }\end{array}$ & $\begin{array}{l}\text { have achieved } \\
\text { (Jing, L. et al., 2015; } \\
\text { Huang Y. et al., 2018a\&b; } \\
\text { Shi, B. et al., 2019) }\end{array}$ \\
\hline Level five & $\begin{array}{l}\text { Under complex } \\
\text { environment (especially } \\
\text { wind, current, wave and } \\
\text { dynamic obstacles), } \\
\text { optimal path planning. }\end{array}$ & $\begin{array}{l}\text { Partial functions have } \\
\text { achieved (Yoo, B., \& } \\
\text { Kim, J., 2016; Sarda, E. } \\
\text { I., et al., 2016; Song, R., } \\
\text { et al., 2017; Ma, Y., et al., } \\
\text { 2018; Singh, Y. et al., } \\
\text { 2018; Wang, N., et al., } \\
\text { 2019) }\end{array}$ \\
\hline Level six & $\begin{array}{l}\text { Realize autonomous } \\
\text { formation and cooperative } \\
\text { control path planning. }\end{array}$ & $\begin{array}{c}\text { Partial functions have } \\
\text { achieved (Ihle, I.A.F. et } \\
\text { al., 2007; Chen, L., et al., } \\
\text { 2019; Ali, H. and Rudy, } \\
\text { R.N. 2019; Shijie, L. et } \\
\text { al., 2019) }\end{array}$ \\
\hline Level seven & $\begin{array}{c}\text { Realizing autonomous } \\
\text { navigation and cognitive } \\
\text { planning in a complex } \\
\text { environment. }\end{array}$ & Not yet achieved \\
\hline Level eight & $\begin{array}{c}\text { Realization of group } \\
\text { USVs game and } \\
\text { autonomous navigation in } \\
\text { complex environment. }\end{array}$ & Not yet achieved \\
\hline
\end{tabular}




\subsection{THE MAIN WORK AND CONTRIBUTION OF THE}

ARTICLE

This paper reviews the researches of path planning based on multi-modality constraint for the USV. Firstly, the research progress of path planning is introduced. Then following a clue of the research progress, the related research methods and classical algorithms are reviewed, especially those applied to the USV, which mainly sorts out the researches related to the USV path planning in the past two decades, analyzes the USV's path planning constraint elements, and divides the USV path planning into three stages from the perspective of multi-modality constraint, and each stage of path planning is analyzed and discussed, which provides a certain reference for the future research in this field (due to the limitation of space, some excellent USVs' path-planning literature are not mentioned in the text, for which we are deeply sorry).

\subsection{THE MAIN STRUCTURE OF THE ARTICLE}

Section 1 of the article introduces the USV's feature and summarizes the whole text. Section 2 mainly analyzes the constraint elements of different modality: planning space, planning time, planning behavior and planning criterion; Sections 3 to 5 divide the USV path planning into three stages based on the modality-constraints elements of the second part: route planning, trajectory planning and motion planning, and analyze the progress and problems of each stage. Section 6 mainly summarizes the whole article and looks forward to the future development of the USV's path planning.

\section{THE PROGRESS OF PATH PLANNING.}

Steven M. LaValle (Steven, 2006) mentioned in his book "Planning Algorithm" that planning is of different meanings in different disciplines. In the field of Robotics, it focuses on the automated mechanical systems which are capable of sensing, driving and computing. The purpose of the planning is to transform the specific tasks that the robot needs to perform (high-dimensional) into the manipulation of the robot (lowdimensional) (Lydia, et al., 2008). In the field of USV Control Theory, one part is to design inputs to physical systems described by differential equations; Another part focuses on the optimal control, namely on the premise of the minimum consumption of the resources (such as time and energy saving), control the system running. And in the field of Artificial Intelligence, the researchers tend to discretize the problem (H. Choset, et al., 2005), which solve the problems like the Rubik's cube or a sliding-tile puzzle that 
needs to build a discrete model, and generally planning time is discrete.

There are several basic ingredients that the USV planning requires: Planning Space, Planning Time, Planning Behavior and Planning Criterion (E. Plaku, et al., 2005).

Planning Space not only refers to the geographic and environmental information, but also contains the dynamic performance of the research objects and other state-space information. Namely, it covers the necessary information during the planning process. The information is fundamental to the design of the planning algorithm. The information of the initial and goal state is particularly important because the planning algorithm is usually a rule that describes the process from the initial state to the goal state.

Planning Time refers to the sequence of decisions that must be made over time. In general, Planning-Time issues not only focus on completing a task within a specific time but more importantly, the planning process must be followed by the correct and reasonable order of execution (i.e., step by step).

Planning Behavior is the actions through which the USVs could reach the goal state from the initial state. In some cases, there are no restrictions on those actions, and the process of planning is ideal. But for most of the time, the actions are restrained by the natural environment and the dynamic performance of the research objects.

Planning Criterion is the desired constraint condition based on Planning Space and Planning Behavior. There are generally two different types of criterion: feasibility and optimality. Feasibility is to find a plan by which the USV could reach the goal state. Optimality is to find an efficient plan for the shortest voyage, the shortest timeconsuming, the lowest fuel-consuming or the hybrid optimization.

USV Path planning problem is a typical problem in planning issues. Path planning problem can be defined as a planning problem with the intention of planning out a path which meets certain requirements. Different modality requirements mean different Planning Criteria (modality refers to a mode of planning). Planning Criterion is related to Planning Space, Planning Time, Planning Behavior and Planning Criterion. So according to the different modality constraints of the basic ingredients, the development of USV path planning has been divided into three stages: Route Planning, Trajectory Planning and Motion Planning.

The progress of Path Planning based on multi-modality constraint is shown in Figure 1. 


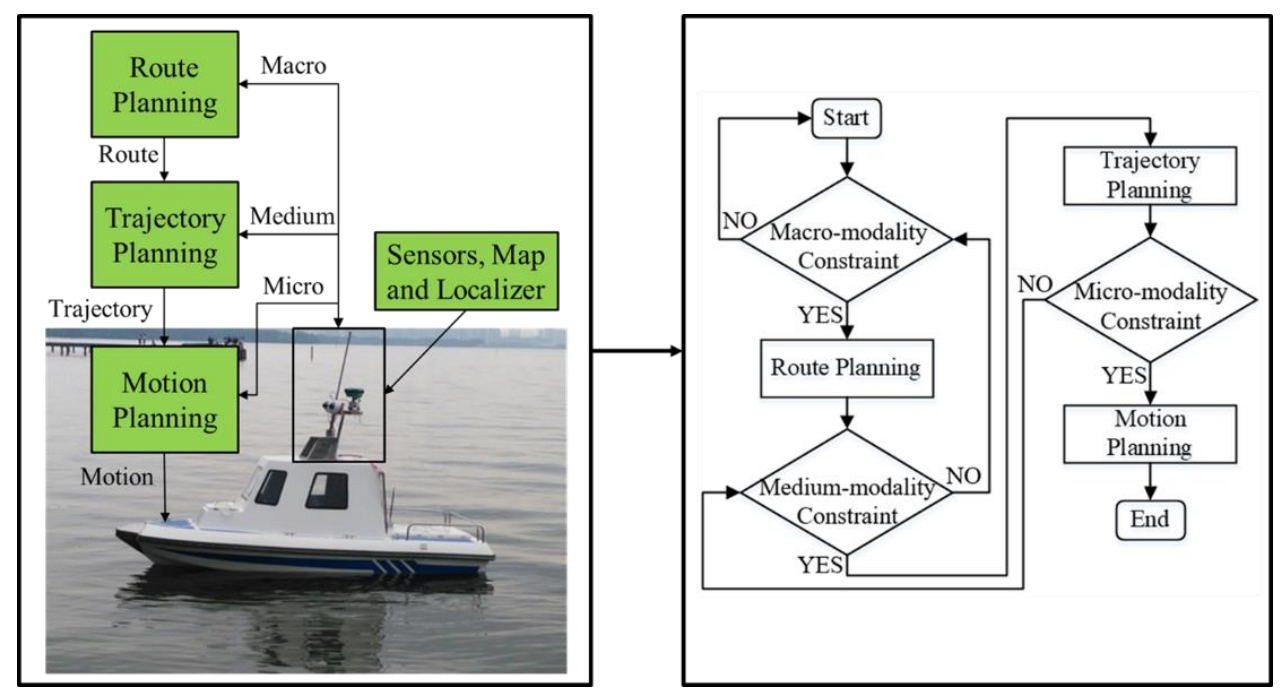

Figure 1 USV Path Planning based on multi-modality constraint

USV Route Planning is an initial stage and is defined as a macro-modality constraint problem. In this stage, the USV is taken as a particle ignoring its scale character and dynamic performance. Planning Space mainly considers the constraints of geography and Planning Behavior is not constrained by the actions of the research object. Planning Criterion focuses on the quality of the path itself. The desired outcome is an optimal route from the starting point to the end point.

USV Trajectory Planning is a transition stage and is defined as a medium-modality constraint problem. In this stage, the Trajectory Planning object takes part of the dynamic constraints into account (such as the shape and kinematics, etc.) and it considers environment constraints of the research object in Planning Space (such as the channel width). Planning Behavior is restricted by the influence of certain part of these constraints (such as linear speed and angular velocity, etc.). Planning Criterion is the improvement for the planned route of the last stage. So the desired outcome in this stage is the optimal trajectory from the starting point to the end point.

USV Motion planning is a final stage and is defined as a micro-modality constraint problem. In this stage, the Motion Planning object is considered with more comprehensive dynamic constraints, including the constraints in Planning Space and Planning Behavior, and it combines path planning with motion control. The desired outcome is a practical motion path from the starting point to the end point.

The ingredients of Path Planning based on multi-modality constraint are shown in Table 4.

Table 4. The ingredients of Path Planning based on multi-modality constraint

\begin{tabular}{|c|c|c|c|}
\hline Ingredients & $\begin{array}{l}\text { USV Route } \\
\text { Planning }\end{array}$ & $\begin{array}{l}\text { USV Trajectory } \\
\text { Planning }\end{array}$ & $\begin{array}{l}\text { USV Motion } \\
\text { Planning }\end{array}$ \\
\hline
\end{tabular}




\begin{tabular}{|c|c|c|c|}
\hline Planning Space & $\begin{array}{c}\text { Environment } \\
\text { Conditions }\end{array}$ & $\begin{array}{c}\text { Environment } \\
\text { Conditions + Part } \\
\text { (Kinematics) } \\
\text { Constraints }\end{array}$ & $\begin{array}{c}\text { Environment } \\
\text { Conditions + fully } \\
\text { Dynamic } \\
\text { (kinematics } \\
\text { +dynamics) } \\
\text { Constraints }\end{array}$ \\
\hline Planning Time & $\begin{array}{c}\text { Sequence of } \\
\text { Decisions } \\
\text { (Discretization) }\end{array}$ & $\begin{array}{c}\text { Sequence of } \\
\text { Decisions } \\
\text { (Discretization) }\end{array}$ & $\begin{array}{c}\text { Sequence of } \\
\text { Decisions } \\
\text { (Discretization) }\end{array}$ \\
\hline Planning Behavior & $\begin{array}{l}\text { No Restrictions } \\
\text { on Research } \\
\text { Objects'Actions }\end{array}$ & $\begin{array}{c}\text { Partial } \\
\text { Restrictions on } \\
\text { Research Objects' } \\
\text { Actions }\end{array}$ & $\begin{array}{l}\text { Full Restrictions } \\
\text { on Research } \\
\text { Objects'Actions }\end{array}$ \\
\hline Planning Criterion & $\begin{array}{c}\text { Plan Out an } \\
\text { Optimal Route }\end{array}$ & $\begin{array}{l}\text { Plan Out an } \\
\text { Optimal } \\
\text { Trajectory }\end{array}$ & $\begin{array}{c}\text { Plan Out an } \\
\text { Optimal Motion } \\
\text { Path }\end{array}$ \\
\hline
\end{tabular}

In term of routing planning, trajectory planning and motion planning, the main difference is that the specific constraint factors are variational. In other words, the specific application scenarios are different.

As for routing planning like the ship's path planning in the large scale area, for example, the routing planning for a ship which will navigate from Hawaii port to Panama port, it does not need to consider full detail about how the ship will reach Panama port. In this stage, we should regard the ship as a particle and ignore other factors such as the specific ship's shape and ship's speed and so on. Figure 2 shows a ship routing planning from Hawaii port to Panama port.

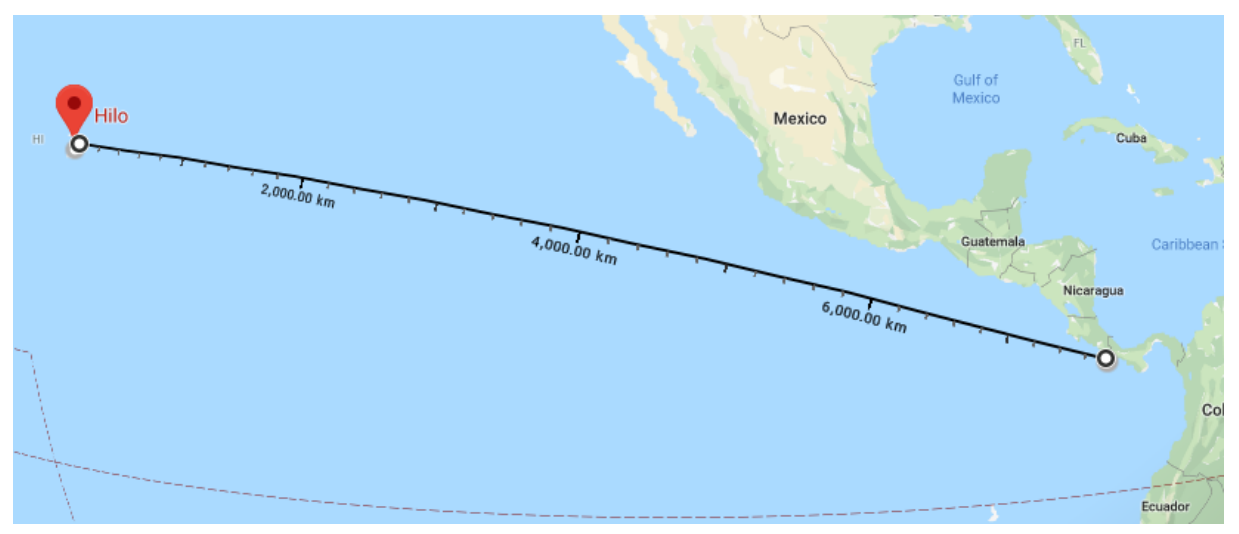

Figure 2 a ship routing planning from Hawaii port to Panama port.

As for trajectory planning like the ship's path planning in the middle scale area, for instance, when the ship reaches Panama port, it needs to think how to enter the inner port, it needs to consider some constraint factors such as specific ship's size and speed 
and so on. In this stage, we should consider some constraints and ignore some unnecessary factors such as moments and forces and so on. Figure 3 shows a ship trajectory planning for how to enter the inner port, the ship is navigated under trajectory planning, in which there are some information that the ship needs to know, such as which entrance is suitable to enter according to the ship sizes, the entrance sizes and its kinematics (e.g. velocity and accelerated velocity etc.).

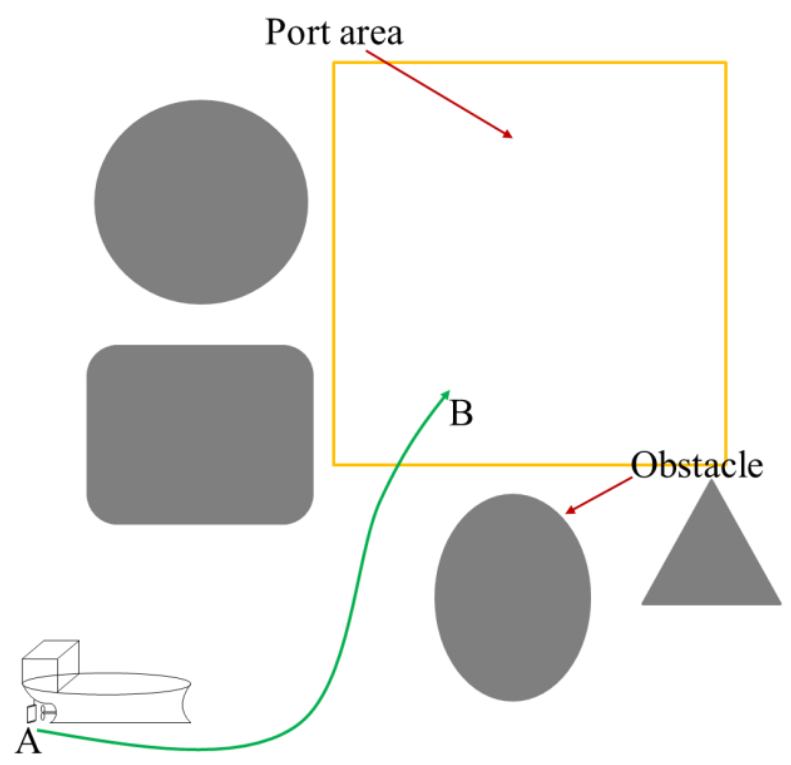

Figure 3 a ship trajectory planning for how to enter the inner port.

As for motion planning like the ship's precise path planning in the small scale area, for example, the motion planning is that a ship berths alongside, which needs to know how to precisely steer to berth. In this stage, the ship is regarded as a rigid body, and we should consider fully constraint factors such as the ship's force and inertia moment and so on. Therefore, the ship's motion planning is applied to more scenarios that require precise tasks such as berth alongside and unberthing operation, etc. Figure 4 shows a ship motion planning for how to berth, the direction of ship at point $\mathrm{A}$ is north 0 degree (the blue arrow at point $\mathrm{A}$ ), the ship needs to know how to steer to reach point $\mathrm{B}$, and keep its direction along the shoreside (blue area) and maintain the direction of blue arrow at point $\mathrm{B}$, in which the ship needs to consider its dynamics (such as inertia forces and moments of the ship's surging, swaying and yawing etc.) and kinematics (such as ship's velocity) and its specific sizes constraints. 


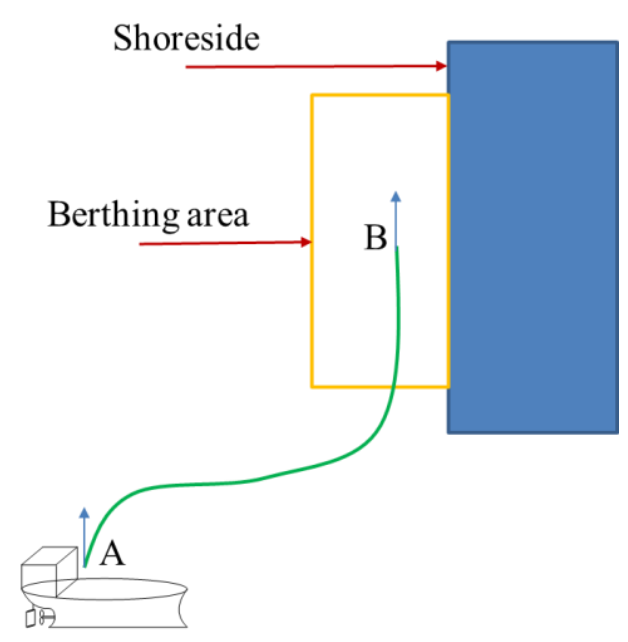

Figure 4 a ship motion planning for how to berth.

Overall, of all the ingredients of USV Path Planning based on the multi-modality constraint, the purpose of the research is to improve the efficiency and effectiveness of the Path Planning. The next three chapters will give specific details of the application scenarios and typical methods for the three stages, respectively. In particular, methods for USV in these three stages are reviewed in detail.

\section{ROUTE PLANNING.}

At the basic stage, Route Planning is defined as a macro-modality constraint problem, that is, its application scenarios are often under large scale environments. In such circumstances, the USV is usually taken as a particle and its dynamic performance is ignored. Route Planning is usually applied to the macro-modality constraint environment, such as the environment shown in Figure 5, in which we do not consider its specific sizes constraints and any dynamic constraints.

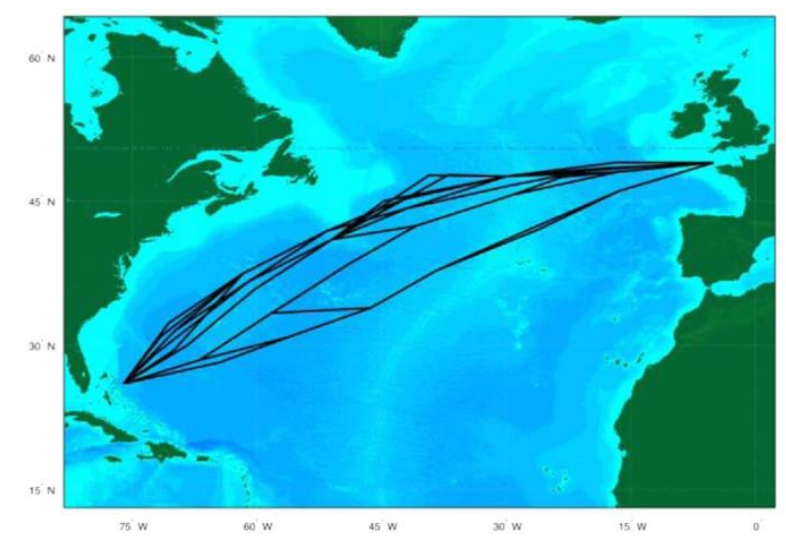

Figure 5 the application in USV Route Planning (Vettor, R., \& Guedes Soares, C.. 2016) 


\subsection{TWO IMPORTANT PARTS OF ROUTE PLANNING}

Route Planning has been studied for many years and many classical algorithms have been proposed. Most of these algorithms take two steps: environment modeling and optimal path searching.

\subsubsection{ENVIRONMENT MODELING}

Environment modeling is the foundation for Route Planning. In this step, not only the planning space is transformed to a geometry space, but also the whole space is discretized into multiple sub-spaces. Visibility Graph, Voronoi Diagram, and grid Map are the common modeling methods (Latip, N. B. A. et al., 2017; Kim, J. et al., 2011; Huajun, L. et al., 2006; Huai Yang. 2016; Chao C., et al., 2014; Candeloro M. et al., 2017; Niu et al., 2019; Yang, J. M. et al., 2015). One of the most widely used ways of environment modeling in Route Planning is Grid Map (Raster Map). This approach decomposes a planning space into many rectangular areas, usually called grids. Figure 6 shows the Visibility Graph, Figure 7 shows the Voronoi Diagram, Figure 8 shows the grid map.

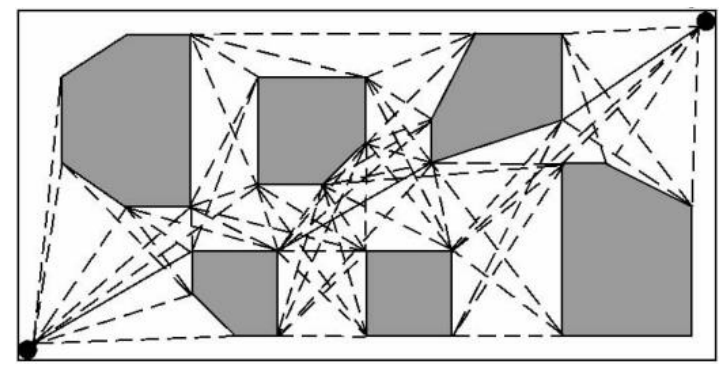

Figure 6 Visibility Graph (Chao C., et al., 2014)

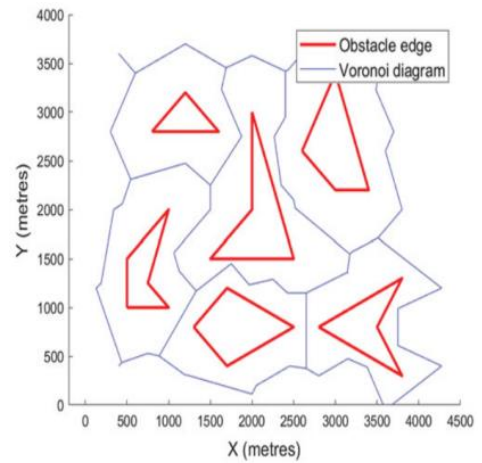

Figure 7 Voronoi roadmap of polygon obstacles (Niu, H., et al., 2019) 


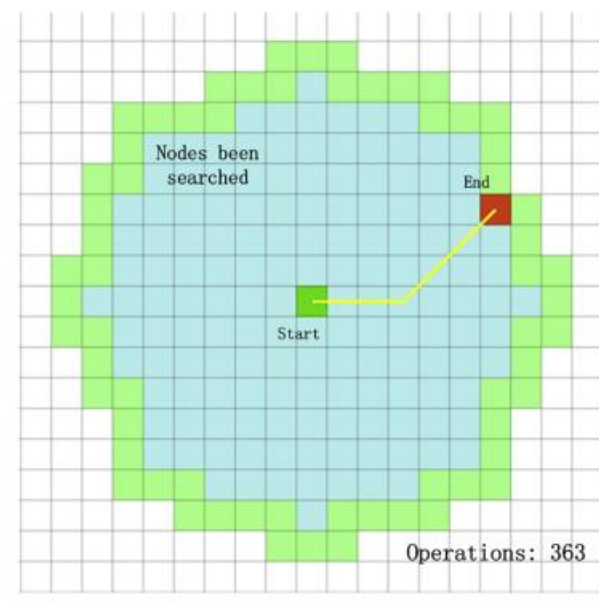

(a)

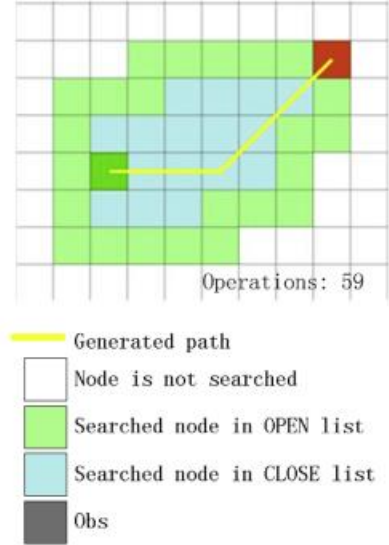

(b)

Figure 8 the Grid Map (Song, R. et al., 2019)

Generally, search-path time, search-path completeness and search-path optimality are the main factors for performance of search-path algorithm (Latip, N. B. A. et al., 2017). The three environment models for performance of search-path algorithm have been discussed according to the above three factors.

(1) search-path time.

As for Visibility Graph method, search-path time of visibility graph method mostly depends on the obstacles, which means search-path time will change according to change of number of obstacles, specifically, it will increase if obstacles of search area change.

As for Voronoi Diagram, generally, the complexity of search-path time can be considered as $O(n \log (n))$, in which $\mathrm{n}$ is the number of the obstacles vertices (Niu et al., 2019), and for Visibility graph method, the complexity of search-path time can be considered as $O\left(n^{2}\right)$ (Ghosh and Mount, 1991).

As for Grid Map, the search-path time will depend on the density of grid map resolution. If the distance between the grid lines is too large, many environment information will not be shown, and an accurate path search cannot be performed. If the distance between the grid lines is too small, and a small distance between the grid lines will lead to the enormous search-path time, meanwhile if the number of grids increase, the searching space might grow enormously (Lei X. et al., 2019).

(2) search-path completeness.

As for Visibility Graph method, the search-path completeness of Visibility Graph method is better compared to Voronoi Diagram and Grid Map, because paths are selected according to the relationship between obstacles. For Voronoi Diagram, because it is expanded by obstacles, there are fewer search nodes, and the number of search nodes and search freedom is less than that of Visibility Graph. For Grid Graph, it is mainly based on the direction of grid to select the path, the path selections are 8 or 
16 directions, so the selection of path points will be more constrained than Visibility Graph and Voronoi Diagram (Zhe Du et al 2018 \& 2019).

(3) search-path optimality.

Generally, for the Visibility Graph, the search path is relatively short, but this path often does not meet the practical requirements, and further optimization is needed. For the Voronoi Diagram, the search path is generally longer than that of the Visibility Graph, but the search path is often farther from the obstacles than that of the Visibility Graph, that is to say, the search path is longer than that of the Visibility Graph, and the path that the Voronoi Diagram get is more secure. For Grid Map, due to the jag effect of Grid Map, the search path of Grid Map is not optimal, but in recent years, there are many scholars that smooth the grid-nodes search, that is to delete unnecessary search nodes to make it smoother and shorter path (Hongwei, W., et al., 2010; Song, R. et al., 2019).

\subsubsection{OPTIMAL PATH SEARCHING}

Optimal path searching algorithms play an important role in macro-modality constraint Route Planning.

Searching algorithms are mainly divided into the heuristic method, evolutionary algorithm (swarm intelligence algorithm) and potential field algorithm. Heuristic algorithms include $A^{*}$ algorithm (Hart, P. et al., 1968) and Lifelong planning A* (Koenig, S., et al., 2004), etc; Evolutionary algorithms include Particle Swarm Optimization (PSO) algorithm (Shi, Y., \& Eberhart, R. C. 2002; Ma, Y et al., 2018; Liang et al., 2019), Genetic Algorithm (GA) (Maulik, U., \& Bandyopadhyay, S. 2002; Qu H. et al., 2013), Ant Colony Optimization algorithm (ACO) (Lazarowska A. 2015), etc; Potential field algorithms include Vector Field (Xu H. et al., 2016), Artificial Potential Field (APF) method (Kohei, 2016), and Fast Marching Method (FMM) (Song et al., 2017), etc.

Heuristic algorithms generally consume more time and memory with the increasing of computing range. At present, although there are many applications which need to use Heuristic algorithms, the algorithms cannot adapt to complex environments well. Evolutionary algorithms can adapt to complex environments, but they may often fall into local optimum; the path of calculation often is stochastic, and the computational time is long. Potential field algorithms are widely used in recent years, but it is also easy to fall into local optimum.

For USVs route planning application, since the dynamical constraints are not considered in this stage, in terms of planning behavior, a USV has no difference from a particle. It can reach somewhere without any posture. In addition, as for planning 
space the application scenarios are usually in large areas, like the open sea. As for planning criteria, the planning approaches for USVs in this stage often combine the classical algorithms mentioned earlier with marine instruments and regulations (as shown in Figure 9) to plan out an optimal route.

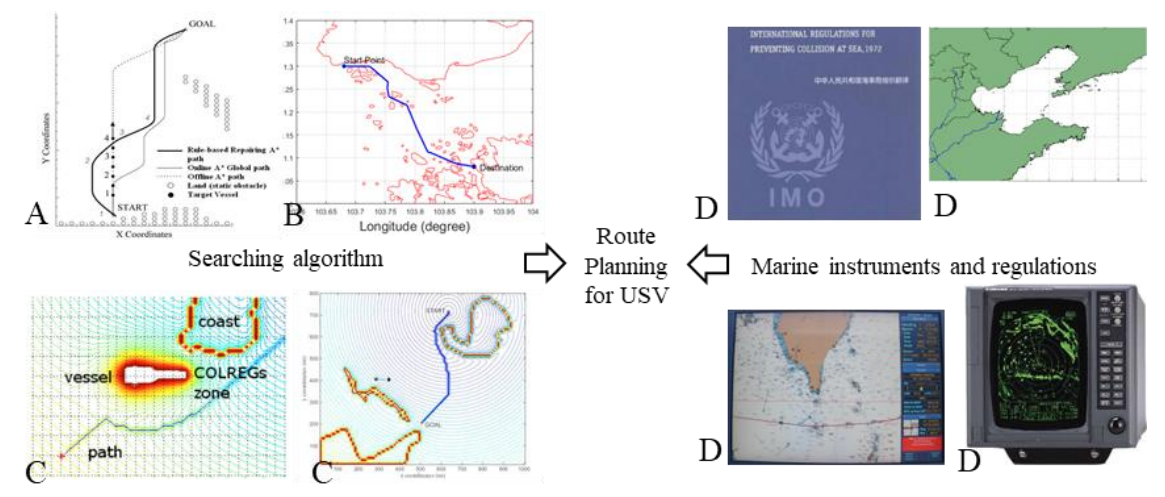

Figure 9 The common way of route planning for USV (A(Campbell, S. \& Naeem, W., 2012), B(Niu, H., et al., 2016), C(Naeem, W., 2016), D (Baidu website gallery))

USV Route planning based on the macro-modality constraint, in terms of routeplanning space, the searching methods' constraints are the same as environmental constraints, it is not necessary for USVs to consider their specific shape, kinematics, and dynamics.

Jiayuan, et al. (Jiayuan, et al., 2011\&2012) combined electronic chart (e-chart) and marine radar with Dijkstra algorithm (Dijkstra, E.W. 1959) to achieve global and local route planning for USVs. They regarded a USV as a particle in the macro-modality environment and used e-charts to get global environment information. And then with the help of the Dijkstra algorithm, they can plan out a global path for the USV. During the voyage, they used a smoothing method to deal with an original radar image and transformed it into a binary image (map) which was used directly for path searching. Finally, with the help of the Dijkstra algorithm, a local path can be planned-out in realtime. Wang Chenbo et al. (Wang Chenbo et al., 2018) realized route planning of a USV in an unknown environment based on reinforcement learning method. This method can achieve better route planning after a certain number of iterations, but it does not consider other environmental factors, such as moving obstacles. Gu, S. et al. (Gu, S. et al., 2019) proposed a route planning method for a USV based on topological location relationship. They constructed the topological map by the topological location relationship, which simplified the search space, and realized the route search of a USV by combining a Dijkstra algorithm. Song, R. et al. (Song, R. et al., 2019) based on the conventional $\mathrm{A}^{*}$ algorithm to carry out the USV search path, by reducing the unnecessary nodes to achieve the smooth path, and its practical ship experiments verified that the smoothing $\mathrm{A}^{*}$ algorithm can reach the target point in a shorter path. 
In terms of route-planning time, planning time is discretization. For example, by making use of e-chart, Jian, L. (Jian, L. 2014) chose the APF method to search a path for a USV in the macro-modality environment. They assumed that there were many small islands and reefs near the shore, thus it is important for a USV to plan out a safe path among these obstacles. Firstly, they marked out the obstacles and feasible zone on an e-chart. And then they improved the repulsive potential to make sure the resultant potential on goal point is the minimum. Finally, they calculated the safest path from the start point to the goal point by using a sequence of decisions.

Lazarowska (Lazarowska, A. 2020) proposed an approach to solving a security problem in routing planning, which utilizes the concept of a discrete APF and a path optimization algorithm, calculates an optimized collision-free route path for a ship. The simulation results show that this method can effectively realize ship route planning in near-real-time.

In terms of route-planning behavior, it has no restriction to research objects' actions. For example, to avoid the collision in the macro-modality constraint environment, the International Marine Collision Regulations (COLREGS) has to be mentioned in the maritime domain (U.S. Department of Homeland Security, 2010). All the ships must obey the rule to prevent collision accident at the sea. The regulations are introduced to normalize the planned path. Researchers (Yanshuang, 2010; Huai Yang, 2016; Campbell, 2012) firstly planned out a global path by A* algorithm in the macromodality constraint environment. And then based on COLREGS, they classified collisions into different situations and worked out different behavior to avoid each situation. The behavior corresponded to different paths. Naeem, et al. (Naeem, et al., 2016) combined COLREGS with modified APF algorithm to generate COLREGScompliant trajectories in the presence of both stationary and dynamic obstacles.

In terms of route-planning criteria, it is important to plan out an optimal route generally. Song et al. (Rui Song, et al., 2017) proposed a novel multi-layered fast marching (MFM) path planning method for unmanned surface vehicle path planning in a time-variant maritime environment. The proposed MFM method to provide a safer and more optimal path by using two operation handlers. The path keeps a safe distance away from obstacles and reduces the energy cost by following counter-flow areas. They considered route planning in the context of macro-modality constraints only, and USV dynamics, moving obstacles and sufficient trajectory smoothness have not been considered in the results. Yuxuan, Z., et al (Yuxuan, Z., et al., 2017) surveyed a coverage path planning algorithm, in which they proposed the dynamic raster method with the heuristic search algorithm to achieve a more reasonable and effective path compared with Yang, S. X. \& Luo, C.'s method (Yang, S. X \& Luo, C. 2004), similarly, they can not consider the kinetics and dynamics of the USV. 


\subsection{SUMMARY OF ROUTE PLANNING}

In summary, there are plenty of sophisticated algorithms proposed in this stage, and most of them can perfectly adapt to the application of USV route planning. But Route Planning is not a very accurate path. As it does not consider the dynamic constraints, it is difficult to apply to some actual scenarios that require more precise path planning.

USV Route Planning can be described as follows:

(1) USV Route Planning problem can be transformed into a reachable problem (R).

(2) USV Route Planning constraints condition:

1) Planning Space: The planning space is only constrained by the connectivity of the environment;

2) Planning Time: Planning time is discrete;

3) Planning Behavior: There are no requirements for planning behavior;

4) Planning Criteria: The planning criterion is to solve an optimal route, focusing only on the accessibility, and not on its own constraints and how to get there.

(3) USV Route Planning Model:

$$
R=\left\{\begin{array}{lc}
\text { connected } & R=1 \\
\text { un-connected } & R=0
\end{array}\right.
$$

$\mathrm{R}=1$ means reachable, and $\mathrm{R}=0$ means unreachable. In other words, the route planning problem can be summarized as reachable or not. Because route planning ignores dynamic factors and its own shape, it simply abstracts the planning problem into a line selection problem from one particle to another.

(4) Route planning based on Macro-modality constraint: scope of application, modality problem, constraint problem, and typical approaches are shown in Table 5.

Table 5. Route planning based on Multi-modality constraint: scope of application, modality problem, constraint problem and typical approaches

\begin{tabular}{|c|c|c|c|}
\hline Scope of application & $\begin{array}{l}\text { Modality } \\
\text { problem }\end{array}$ & $\begin{array}{l}\text { Constraint } \\
\text { problem }\end{array}$ & $\begin{array}{c}\text { Typical } \\
\text { approaches }\end{array}$ \\
\hline $\begin{array}{l}\text { In the macro-modality } \\
\text { constraint environment } \\
\text { like RPG (Role-playing } \\
\text { game), vehicle } \\
\text { navigation in city, } \\
\text { indoor navigation for }\end{array}$ & $\begin{array}{c}\text { Macro-modality } \\
\text { problem }\end{array}$ & $\begin{array}{l}\text { No kinematics } \\
\text { and dynamics } \\
\text { constraint } \\
\text { problem, } \\
\text { research object } \\
\text { as a particle }\end{array}$ & $\begin{array}{l}\text { Dijkstra algorithm, } \\
\text { A* algorithm, APF, } \\
\text { PSO algorithm, GA, } \\
\text { ACO algorithm, } \\
\text { FMM, etc. }\end{array}$ \\
\hline
\end{tabular}


robots, and ship routing,

etc.

\section{TRAJECTORY PLANNING.}

Along with the clues, some scholars begin to pay closer attention to the dynamic constraints of the research object itself. When considering a research object in a physical system, the planning path cannot be as ideal as shown in Figure 5. Hence the study of Trajectory Planning is of vital importance.

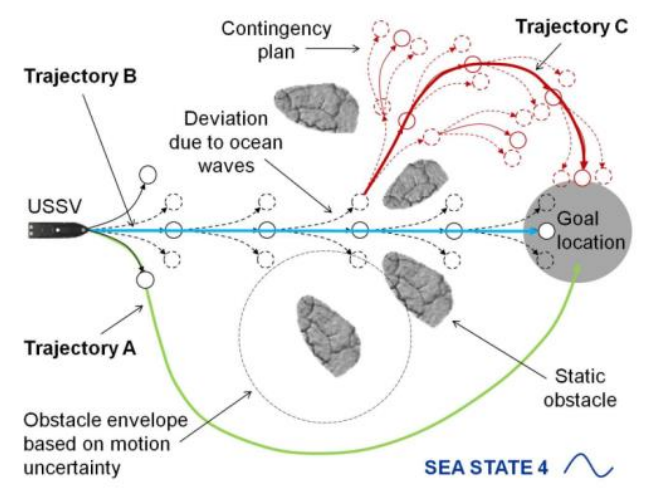

Figure 10 The application in USV Trajectory Planning (Svec, P. et al., 2011)

Trajectory planning can be seen as an improvement of Route Planning. At this stage, Trajectory planning is defined as a medium-modality constraint problem. Instead of regarding a research object as a particle, this stage begins to consider its part dynamic constraints, like size, speed, heading, curvature. In other words, what the Trajectory Planning wants to do is to make the planning path smooth and continuous, and conforms to one or a few of the physical characteristics of the research object (as shown in Figure $10)$.

\subsection{TRAJECTORY PLANNING ANALYSIS}

The common approaches in this stage are classified into two types, curve fitting and multi-constraint optimization (as shown in Figure 11). 


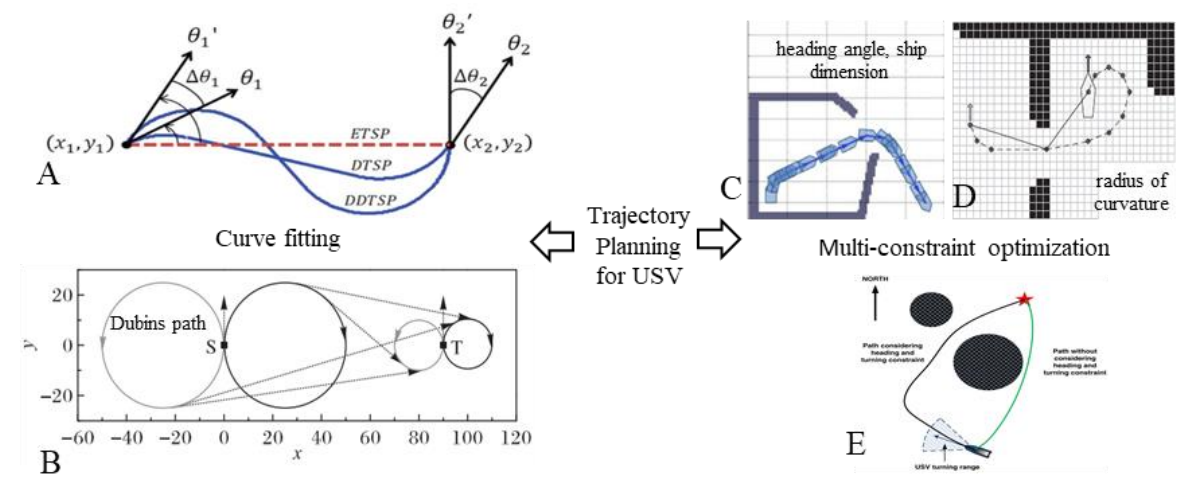

Figure 11 Two approaches of trajectory planning for USV (A (Cohen, L., et al., 2017), B (Liu, L., et al., 2017), C(Yang, J. M., et al., 2015), D(Kim, H., et al., 2014), E(Liu, Y. \& Bucknall, R. 2016))

Curve fitting is a way that constructs a curve to make the best fit to the certain polyline. For a USV, it is a way to find the best-fitted curve for the planned path. In terms of trajectory-planning space and behavior, it considers environment conditions and the part of USV dynamic constraints. For example the curve should meet the constraint of the minimum turning radius. It makes the planned path smoother and more continuous in a medium-modality constraint environment. A common approach is a Dubins path (F. Dougherty, et al., 1990). The idea of this method is to find the shortest possible path for a particle with unity speed that meets a minimum curvature bound between a starting pose (which contains position and orientation) and a finishing pose consisting of at most three pieces, and each piece is either a straight line or an arc of a circle. The concrete path composition can be "CLC", "CCL" or "CCC" ("C" and "L" stand for circle and line respectively) (Anderson, et al., 2014).

This method was first applied in UAVs, but in recent years, there are also some applications of Dubins path in USV trajectory planning (Yi Chen, 2016; Liu L. et al., 2017). For example, Yi Chen (Yi Chen, 2016) considered a turning process in the actual sailing of the USV in the medium-modality constraint environment. He combined the "CLC" Dubins path with a genetic algorithm to solve the path optimization problem of the pose (positions and heading angles) transition from the starting point to the end point. However, the Dubins path does not result in curvature continuous paths due to that the curvature of a straight line is 0 , whereas a circle arc has a certain curvature. Hence, there will be a jump in the curvature from 0 to a certain value when moving from the straight line to the circle arc. To overcome the shortcoming, some scholars replaced circular arcs to some special curves in the medium-modality constraint environment, like Fermat's spiral (FS) (Dahl A. R., 2013), B splines (Hong Li, et al. 2016) and Bessel curve (Yi Wang, et al. 2012). These curves are good means to connect successive straight lines, because the curvatures of lines are equal to zero at the origin, a property which makes lines suitable for being connected into a straight line without inducing curvature discontinuities. Xiaojie Sun (Xiaojie Sun, 2016) used floating-point 
numbers and the turning radius of KT equations as the order and the radius of curvature of Bessel curve to restrain the path, and finally got a smooth and continuous trajectory for USVs. Wang, N., et al. (Wang, N., et al., 2019) proposed a multilayer path planning algorithm to search path in complex marine environments. In which they B-Spline method to minimize yaw-cost for the USV path planning.

Multi-constraint optimization is more direct in the medium-modality constraint environment. The main idea is to add part dynamic constraints to the original route planning algorithm. It prompts the planned path more practical and reasonable. For example, Kim, M., et al. (Kim, M., et al., 2014) added a heading angle and radius of curvature constraints to optimize the path. They took into account the possible USV's steering heading angles in the process of planning, turning the original two-dimensional $(x, y)$ planning space into three-dimensional $(x, y, \theta)$. At the same time, they improved the $\mathrm{A}^{*}$ algorithm by adding the radius of curvature into actual cost function $\mathrm{g}(\mathrm{x})$ to make turning process more reasonable and effective. In the medium-modality constraint environment, they focused on considering the adequacy of planning space. Liang $\mathrm{Hu}$, et al. (Liang $\mathrm{Hu}$, et al., 2019) proposed a new on-line path planning method for USVs which generates collision-free and COLREGs-compliant paths using a multiobjective optimization approach based on the particle swarm optimization. The proposed method determined the type of encounter and whether a target ship complied with the COLREGs. Therefore, in the medium-modality constraint environment, it is more concerned about the adequacy of planning behavior, such as considering part restrictions in research objects' actions (such as the velocity, heading course, etc.).

In addition, Yang J. M., et al. (Yang, J. M., et al., 2015) considered the ship's initial heading and its size constraints. Firstly, they proposed the Finite $A^{*}$ algorithm by improving $A^{*}$ algorithm to get a shorter path compared with post- $A^{*}$ algorithm (Botea, A. et al., 2004) and A* algorithm, and then they considered the ship's initial heading and size to achieve a safer and smoother path. Others (Zuquan, et al., 2015) focused on the constraint of speed to avoid dynamic obstacles. Firstly they used the Particle Swarm Optimization algorithm (PSO) to plan a path in a static environment. And then they made use of relative speed between the USV and dynamic obstacle to find a navigable path. Moreover, in the medium-modality constraint environment, $\mathrm{He}$, Y., et al. (He, Y., et al., 2017) proposed quantitative analysis of COLREG rules and seamanship for autonomous collision avoidance at open sea. They presented a situation identifying the model and defined four distinct stages in the entire encounter process for autonomous collision avoidance, in which appropriate collision avoidance actions can be taken according to different situations and stages, and they also built quantitative computing models for first time-in-point of the close-quarters situation and first timein-point of immediate danger. Rong, H., et al. (Rong, H., et al., 2019) investigated the 
uncertainty of ship trajectory prediction via a probabilistic prediction model. In which they established a parameters of the probabilistic models according to historic ship trajectory data that is from Automatic Identifcation System (AIS). Chen C., et al., (Chen, C., et al., 2019) proposed a trajectory planning algorithm that considered USV kinematics equation in the planning process. In which they utilized the Q-learning (Watkins \& Dayan, 1992) and USV features to achieve a better path compared A* algorithm, RRT (Rapid-exploring Random Tree) algorithm. However, they did not consider the dynamic obstacles and ship collision avoidance rules in the planning area, furthermore, the Q-learning algorithm that they used may be too simple and restricted compared other artificial intelligence algorithms such as deep deterministic policy gradient algorithm (Lillicrap, T. P. , et al., 2015) and distributed proximal policy optimization algorithm (Heess, N., et al., 2017).

\subsection{SUMMARY OF TRAJECTORY PLANNING}

In the trajectory planning stage, the planning space, environment conditions and part of dynamic constraints are considered, such as dubins method considering environmental accessibility and shape of an object, etc. As for planning time, it is a sequence of decisions. As for planning behavior, only part restrictions in research objects' actions, such as curve fitting and multi-constraint optimization take into account speed and turning radius of the object, etc. As for planning criteria, it is necessary to plan out an optimal trajectory.

Therefore, Trajectory Planning problem can be described as follows:

(1) USV Trajectory Planning problem can be transformed into a reachable problem $\left(\mathrm{R}^{\prime}\right)$.

(2) The constraint conditions of USV Trajectory Planning are as follows:

1) Planning Space: The planning space has environmental connectivity constraints and partial self-size and part dynamic constraints;

2) Planning Time: Planning time is discrete;

3) Planning Behavior: there are some planning behavior requirements, such as speed, heading, etc;

4) Planning Criterion: The planning criterion is to optimize the trajectory, focusing on whether the trajectory can meet both the environmental connectivity and some of the USV's static(size, shape, etc.) and part dynamic constraints.

(3) USV Trajectory Planning Model:

$$
R^{\prime}=\left\{\begin{array}{lll}
\text { part of constraint } & \text { connected } & R^{\prime}=1 \\
\text { part of constraint } & \text { un-connected } & R^{\prime}=0
\end{array}\right.
$$


$\mathrm{R}^{\prime}=1$ means reachable, and $\mathrm{R}^{\prime}=0$ means unreachable. The problem of trajectory planning can be summarized as the issue of reachability under partial constraints. Because the trajectory planning only considers part of the dynamic and shape constraints, the problem of the trajectory planning can be seen as a problem that a shaped object can reach from one location to another.

(4) Trajectory planning based on Multi-modality constraint involving the scope of application, modality problem, constraint problem, and typical approaches is shown in Table 6.

Table 6. Trajectory planning based on Multi-modality constraint: scope of application, modality problem, constraint problem and typical approaches

\begin{tabular}{cccc}
\hline Scope of application & $\begin{array}{c}\text { Modality } \\
\text { problem }\end{array}$ & $\begin{array}{c}\text { Constraint } \\
\text { problem }\end{array}$ & $\begin{array}{c}\text { Typical } \\
\text { approaches }\end{array}$ \\
\hline & & $\begin{array}{c}\text { Part of dynamic } \\
\text { constraint (i.e. } \\
\text { shape+kinematics }\end{array}$ & $\begin{array}{c}\text { Dubins approaches } \\
\text { and related } \\
\text { improvement } \\
\text { approaches, Fermat's } \\
\text { In the medium - }\end{array}$ \\
modality constraint & Medium- & constraint) & spiral, B splines, \\
environment USVs, & modality & problems, like & Bessel curve, PSO \\
UGVs, UAVs, etc. & problem & size, speed, & algorithm, A* \\
& & heading, curvature & algorithm, Dijkstra \\
& & and etc & algorithm, etc. \\
\hline
\end{tabular}

Although trajectory planning makes planning path better and closer to the real trajectory, the part dynamic constraints, in fact, are independent of each other and not full enough. Because those constraints are considered separately, which means one or two of them are being added linearly into the algorithm, but the interactions between them are neglected. In addition, trajectory planning does not really achieve the final goal ("How should I do to get there") of path planning for the USV. How to achieve the final goal will be addressed in the next stage.

\section{MOTION PLANNING.}

Motion Planning is the final stage and the final goal for path planning. At this stage, Motion planning is defined as a micro-modality constraint problem. It can be seen as fine control for a research object, which focuses on whether the planned path can be found via its own control system. To achieve this goal, the dynamic characteristics of the research object should be studied deeply. But unlike the Trajectory Planning which only needs to consider one or two dynamic constraints separately, Motion Planning 
must treat a research object as a rigid body. That means all the dynamic constraints should be considered. Thus, it is important to study the mathematical model in this stage.

\subsection{TWO SYSTEMS OF MOTION PLANNING}

According to the control ability, there are two kinds of research objects in this stage: the object of fully actuated system, like the industrial robot and mechanical arm; and the object of underactuated system, like an USV (Figure 12 shows an application in USV Motion Planning).

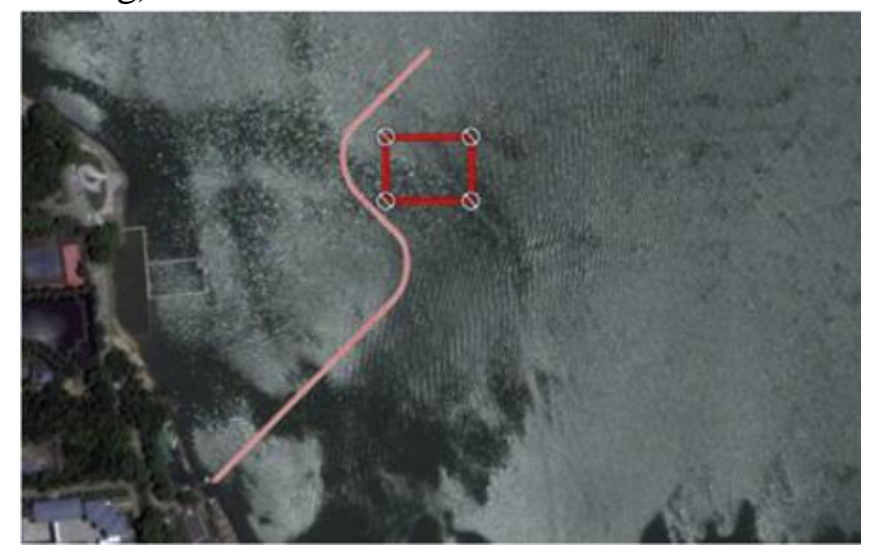

Figure 12 The application in USV Motion Planning (Zhe Du et al., 2018)

For a fully actuated system, the degree of control is equal to the degree of freedom. All the motion poses can be controlled precisely by dynamic equations. As to Motion Planning, the only thing needed to do is to adjust and optimize the control parameters to realize different paths for different tasks. Thus, it is easy to deal with the problem when the research objects are fully actuated system. However, for an underactuated system, because the degree of control of the underactuated system is less than the degree of freedom, not all the motion state can be achieved. Thus, for an underactuated system, one of the important tasks of motion planning is to figure out which state the research object can reach.

\subsection{TWO TYPES OF MOTION PLANNING}

In general, there are two kinds of methods to solve motion planning problems for the underactuated systems such as USVs. One is based on Control Theory, and the other is based on random sampling.

\subsubsection{BASED ON CONTROL THEORY}

The first one is based on Control Theory. The approach is divided into two steps 
in the micro-modality constraint environment. Firstly, by making use of traditional path planning (route planning) algorithm, a collision-free route from the start point to the end point is planned out. Then, based on the mathematical model of a research object, a controller is designed, which contains kinematics and dynamics constraints. Taking advantage of the motion controller, the final path is produced by the second planning (Liang Kang, 2010).

The key point of this approach is motion control that uses control strategy to reconstruct or approximate the "ideal path" which is planned for the first time. With different goals, the motion control can be classified into point stabilization, path tracking and path following (Hao Yu. 2014). Although this approach can be used to solve the problem to a certain degree, the way of solving belongs to the field of control rather than the field of planning in the micro-modality constraint environment.

For example, Escario et al. (Jose B. Escario, et al., 2012) studied the optimization of USV motion planning by applying Ant Colony Optimization metaheuristic. The aim of their work is to find optimal maneuvers for an USV by using an ant algorithm. They modified the original ACO algorithm and the algorithm gave an optimal path in the micro-modality constraint environment. Ma et al. (Ma, Y., et al., 2018) studied the multi-objective path planning problem for USVs in environments with currents effects. They focused on path planning problem that tends to simultaneously optimize four objectives (the path length, the path smoothness, the economic cost, and the path safety) and at the same time it is subject to the collision avoidance, motion boundaries and currents effects in the micro-modality constraint environment. It takes dynamic behavior into account and gives an optimal motion planning.

Moreover, Zhe Du et al. (Zhe Du et al., 2018) established trajectory units based on ship mathematics which is Maneuvering Mathematical Group (MMG) model (Ogawa, O., 1977), and considered the dynamics requirements of a USV, and then proposed a motion planning algorithm for an USV. Based on the literature (Zhe Du et al., 2018), Zhe Du et al. (Zhe Du et al., 2019) further proposed the 32 Directional USV trajectory units and a new path search algorithm for the USV motion planning. Bitar, G., et al. (Bitar, G., et al., 2019) considered a warm-started optimized motion planning algorithm for USVs. Firstly, they harnessed A* algorithm to get shortest piecewise linear path, but the kind of path is not feasible for USV motion planning; Secondly, they connected the waypoints that are acquired by $\mathrm{A}^{*}$ algorithm using circles and adds artificial dynamics, to make the path become closer to feasible and practical path. However, they may lose the optimality when modifying the shortest waypoints. 


\subsubsection{BASED ON RANDOM SAMPLING}

Another approach is based on Random Sampling in the micro-modality constraint environment. All the methods in Route Planning stage plan out a deterministic result. That is to say, in the same planning space, the results of any two kinds of planning are the same. However, the dimension (constraints) of the motion planning is much higher than that of route planning, and with the dimension increasing the complexity of the planning problem grows exponentially (NP-hard problem (Canny, 1998)). Some scholars proposed Random Sampling based on motion planning algorithms to solve the problems in the micro-modality constraint environment. The biggest characteristic of these methods results in that any two kinds of planning are different, even in the same planning space. Probabilistic Roadmap Method (PRM) and Rapidly-exploring Random Tree (RRT) are the typical ones.

Firstly, PRM algorithm is proposed by KavrakiL et al. (KavrakiL, et al., 1994) and the algorithm considers all restrictions in research objects' actions. The main idea is to generate random road signs (state) in the space to determine the feasible region (free space), then connect the adjacent road signs to become random roadmap, and finally find out a feasible path on this random roadmap. The nodes on the map represent the pose of the research object, and the connecting lines between those nodes (the edges in the graph) represent the feasible path between different poses. Similar to this idea, the State Lattice (M. Pivtoraiko et al., 2005) is a discretization from the configuration space to a set of states, representing configurations, and connections between these states, where every connection represents a feasible path.

To move a step forward, RRT algorithm is more applicable to an optimal controlrelated problem and a nonholonomic system (Nan, 2014), and the algorithm considers all the dynamic constraints in the micro-modality constraint environment. The main idea is that by corresponding input of control, randomly generates a search tree from the initial state. Each vertex represents a state and each directed edge represents a control input. With the tree growing, the state updates constantly. When a vertex reaches the desired target area, the tree stops growing (S. M. LaValle, 1998). Because the state transition in RRT algorithm can be improved by kinetic models and new node generation can be constrained by kinetic equations, it can be combined with the mathematical model to solve motion planning issues in the micro-modality constraint environment (Na, et al., 2011; Mingbo, et al., 2015; Jinze Song et al., 2010). A popular research direction currently is to apply the RRT algorithm to the dynamic environment. The guidance factor is introduced to the direction of node expansion to enhance the purpose of the search tree's growing (Guangzhen et al., 2013; Heoncheol et al., 2012). Approaches based on Random Sampling are widely used in motion planning of 
the robotics (such as industrial robots, UAVs, etc.). However, like inertia, resistance and response time on waters are larger than those on the ground, the motion control for USVs is far more complex and difficult than UGVs or UAVs, and the above methods of motion planning need to be improved for USVs motion planning. Moreover, with the increasing density of water traffic, the scope of the sailing area is decreasing gradually, and where the USVs need to be more precisely steered.

\subsection{SUMMARY OF MOTION PLANNING}

In the motion planning stage, the planning space, environment conditions and dynamic constraints are considered. For example, control theory and random sampling consider environmental accessibility, the shape of object, kinematics and dynamics of an object, etc. As for planning time, it is a sequence of decisions. As for planning behavior, restrictions in research objects' actions, such as speed, distance, moment and inertia of the object, etc. are considered. As for planning criterion, it is normally necessary to plan out an optimal motion planning.

Therefore, the Motion Planning problem can be described as follows:

(1) USV Motion Planning problem can be transformed into a reachable problem ( $\mathrm{R}^{\prime \prime)}$.

(2) USV Motion Planning constraints condition:

1) Planning Space: The planning space has environmental connectivity constraints and its own size and dynamic constraints;

2) Planning Time: Planning time is discrete;

3) Planning Behavior: Planning behavior requires comprehensiveness, not only to pay attention to when to give more power but also to pay attention to when and how many rudder angles are appropriate;

4) Planning Criterion: The planning criterion is to solve a path containing specific behavioral instructions, focusing on how to navigate to reach the target point, not only depending on the environmental connectivity, but also on how to navigate under the USV dynamics constraints and their own size constraints.

(3) USV Motion Planning Model:

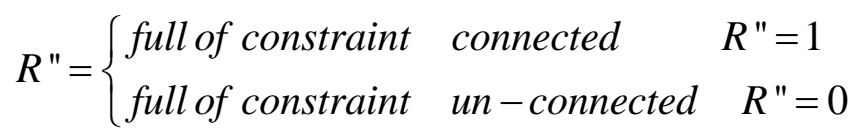

$\mathrm{R}^{\prime \prime}=1$ means reachable, and $\mathrm{R} "=0$ means unreachable. The problem of motion planning can be summarized as the problem of reachable under the constraints of dynamics and its own shape and size etc. Because motion planning considers dynamics and shape size constraints, etc., the USV motion planning is considered as an object 
with size constraints and dynamic constraints, and how to operate from one location to another location.

(4) Motion planning based on Multi-modality constraint including the scope of application, modality problem, constraint problem, and typical approaches is shown in Table 7.

Table 7. Motion planning based on Multi-modality constraint: scope of application, modality problem, constraint problem, and typical approaches

\begin{tabular}{|c|c|c|c|}
\hline Scope of application & $\begin{array}{l}\text { Modality } \\
\text { problem }\end{array}$ & Constraint problem & $\begin{array}{c}\text { Typical } \\
\text { approaches }\end{array}$ \\
\hline $\begin{array}{l}\text { In the micro-modality } \\
\text { constraint environment } \\
\text { the industrial robot, } \\
\text { mechanical arm, UGV, } \\
\text { UAV, USV and etc }\end{array}$ & $\begin{array}{c}\text { Micro-modality } \\
\text { problem }\end{array}$ & $\begin{array}{c}\text { Fully dynamic } \\
\text { constraint (i.e. } \\
\text { shape+ kinematics } \\
\text { +Dynamics } \\
\text { constraint) } \\
\text { problem }\end{array}$ & $\begin{array}{l}\text { A* algorithm, } \\
\text { PRM algorithm, } \\
\text { dijkstra algorithm, } \\
\text { PSO algorithm, } \\
\text { ACO algorithm, } \\
\text { RRT algorithm etc. }\end{array}$ \\
\hline
\end{tabular}

In some special navigation environment, a small range of autonomous control and fine operation becomes necessary, such as the task of self-avoidance and self-berthing in harbor areas. Svec et al. (P. Svec, et al., 2012) have made some attempts. They took advantage of the probability prediction method to solve the problem. They first used a USV mathematical model to predict all the possible trajectories, and then employed min-max game-tree search to select the trajectory which had the smallest collision probability from the possible trajectories pool. But it is still unclear how these possible trajectories are generated (or what the special properties they have) and how to control an USV (the specific instruction) to achieve the planned path.

\section{CONCLUSION AND OUTLOOK.}

This paper focuses on path planning for USV based on the multi-modality constraint. With the research development as a clue, the paper introduced the common approaches and algorithms in three stages: Route Planning, Trajectory Planning and Motion Planning.

(1) Route Planning is a basic stage and Route Planning problem is a macromodality constraint problem. It treats a USV as a particle. So the approaches in this stage often combine the classic algorithms used in USV areas with marine instrument and regulations.

After the above-mentioned summary of the existing technologies of USV route 
planning based on macro-modality constraint environment, the combination of navigation rules, semantic rules and route planning are rarely studied. Those ways could help achieve smarter USVs.

(2) Trajectory Planning is a transition stage and Trajectory Planning problem is a medium-modality constraint problem. It considers the part dynamic constraints (such shape and kinematics) of USVs. The methods in the stage usually have two types: one is curve fitting and the other is multi-constraint optimization. Curve fitting is a way to construct a curve to make the best fit for the planned route path (in route planning). Multi-constraint optimization is more direct in that it improves the original planning algorithm by adding kinematic constraints to make the final path to meet these constraints. The former makes the planned path smoother, while the latter makes it more reasonable.

After the above-mentioned summary of the existing technology of USV trajectory planning based on medium-modality constraint environment, there are still some things to do to realize real-time trajectory planning.

(3) Motion Planning is the final stage and Motion Planning problem is a micromodality constraint problem. Motion Planning is the ultimate goal of path planning for USVs. It treats an USV as a rigid body and focuses on motion control. Thus the approaches in this stage link with control theory and motion prediction. But there is no effective way to guide a USV how to command the control systems to make it get to the goal all by itself. Therefore, there is still much work to do.

According to the above summary of the existing technologies of USV motion planning based on micro-modality constraint environment, full autonomous USV motion planning has not yet been achieved.

In addition, the main challenges of path planning are different, because planning demands in each stage are different.

As for an USV or ship's routing planning, its application scenarios are often a macro-modality constraint in large scale area. For example, it is a path planning from a port to another port, of which the main challenges are weather change, tsunami and typhoon, etc.

As for an USV or ship's trajectory planning, its application scenarios are often medium -modality constraint in medium scale area. For example, the path planning is how to navigate a ship into a port, of which the main challenges are how to avoid the obstacles, including static obstacles and dynamic obstacles.

As for an USV or ship's motion planning, its application scenarios are often precise operations which need micro-modality constraint in small scale area. For example, the path planning is how to maneuver a ship to berth alongside, of which the main challenges are how to steer stably and avoid wind, wave and current effects, 
furthermore, to prevent the ship's drift which has a negative impact on ship's tasks.

Therefore, looking forward to the future development of USVs path planning, we need to proceed from the following directions to help achieve smarter and safer USVs: the multi-element integration of perception system, steady and robust control system, intelligent decision-making system, diversified task execution and complex environment adaptation.

In general, the development of path planning for USVs, which still needs to combine with interdisciplinary approaches to solve problems such as the berthing problem and the path-planning problems for USVs under complex sea conditions (wind, wave, current and weather interference, etc.), which still need to be further solved. In order to solve the above problems, it is necessary not only to consider the actual hydrodynamic influence of USVs, but also to find a reasonable solution to the N-P difficult problem (Michael RG and Johnson DS, 1979; Liu, S. et al., 2011; Ma, Y. et al., 2018). For example, the planned path should consider the purposes that are the smoothness of rudder, the distance of navigation, the economy of navigation and the safety of navigation, etc.

In conclusion, for the short-term and long-term goals of the development of USVs path planning, we should start from the following directions:

1. Short-term objectives:

(1) Quantitative rules and path planning.

The navigation of the USV needs to conform to the navigation rules, and the rules should be quantified to further make the navigation of the USV conform to the collision avoidance rules well. At present, some scholars have made some achievements in this field, such as Zhuang, J. Y., et al. (Zhuang, J. Y., et al., 2011) and He, Y., et al. (He, Y., et al., 2017) etc.

(2) Semantic situation and path planning.

In the future, it is necessary to research the effect of space semantic situation (Zhang, Y., et al., 2018; Wen, Y., et al., 2019) on USV path planning. At present, semantic planning and control are rarely seen in the path planning of USVs, but some scholars have made some research in the mission planning of unmanned underwater vehicles (Patron, P. 2010) and path planning of ground mobile robots (Wang, C. et al., 2019; Yaagoubi, R., et al., 2020). Because the semantic environment can greatly reduce the reliance on complex multi-source data, and greatly reduce the amount of calculation, and then improve the efficiency of calculation.

(3) Advanced control methods and path planning.

The navigation process of USVs is a nonlinear control and uncertain process, the navigation control of USVs in the future needs to combine with advanced control methods. Generally, the control theory is far from practical application, especially there 
are fewer advanced theoretical control methods that are applied for USVs, such as nonlinear control based on manifold space (Sakamoto, N. 2013) and partial differential equations control (Zheng, J. \& Zhu, G. 2019) and so on.

(4) Path planning under complex sea conditions.

It is necessary to propose methods which plan the route and motion commands for USVs in real time under complex sea conditions. In this respect, some scholars have made some researches, such as Song, R., et al. (Song, R., et al., 2017), Ma, Y., et al. (Ma, Y., et al., 2018) and Wang, N., et al. (Wang, N., et al., 2019), etc.

\section{Long-term objectives:}

(1) USVs autonomous berthing.

At present, the intelligent level of USVs has been improved. However, as for autonomous berthing, especially under the influence of current, wind and shore wave effect, is still difficult to achieve. Therefore, it is necessary to research the effects of current, wind and shore wave on autonomous berthing. Some scholars have done some research on this topic, such as Djouani \& Hamam (Djouani, K., \& Hamam, Y. 1995),Okazaki, T., et al. (Okazaki, T., et al., 2000), Meyer, P. J., et al. (Meyer, P. J., et al., 2019) and Martinsen, A. B., et al. (Martinsen, A. B., et al., 2019).

(2) USVs formation coordinated motion planning control.

It is an inevitable trend for the coordinated motion planning control of USVs in the future, because it is difficult to complete complex tasks by relying on a single USV, and the research on the coordinated control of multiple USVs is a key point for the development of USVs formation in the future. Liu, B. et al. (Liu, B. et al., 2019), Chen, L., et al. (Chen, L., et al., 2019) and Li, S., et al. (Li, S., et al., 2019) etc. have done some researches in this area.

(3) Realization of group USVs autonomous navigation in complex environment.

At present, the motion planning and cooperative control of the USV formation are generally carried out in a relatively simple environment, without enough consideration of complex environment, such as strong wind, strong current, waves, multiple moving obstacles, etc., so how to ensure the USV formation to carry out adaptive, efficient and safe navigation task is still a problem to be solved.

(4) USV formation, UAV formation and submarine formation cooperate with motion planning and mission planning control to achieve multi-USVs, multi-UAVs and multi-submarines cooperation to perform complex target tasks.

It is very helpful for the implementation of complex tasks in the future to research multiple robotics and multiple formations for air-sea cooperative motion planning and task planning. For example, in order to deal with oil pollution at sea, it is necessary to monitor oil diffusion in the air and under the sea by multiple UAVs and submarines 
respectively, and to clean up oil pollution by USV formation. Hence, we should develop the air-sea cooperative mission, especially the motion control and collaborative planning of a variety of UAVs, USVs and submarines, and establish a variety of vehicle control systems.

\section{ACKNOWLEDGEMENT}

This work is supported by the National Key R\&D Program of China (No. 2018YFC1407405, 2018YFC0213904); the National Natural Science Foundation of China (No. 51679180, 51579204, 41801375 , 51709218); the Funding of the State Key Laboratory of Surveying, Mapping, Remote Sensing and Information Engineering of Wuhan University (No. 17I03); the Natural Science Foundation of Hubei Province (No. 2016CFB362). The authors are grateful to the three anonymous reviewers for their constructive comments.

\section{REFERENCE}

A. J. Shafer et al., (2008). Autonomous cooperation of heterogeneous platforms for seabased search tasks. 2008 OCEANS, Quebec City, QC, 2008, pp. 1-10.

Anderson, R.P., \& Milutinovic, D., (2014). A stochastic approach to dubins vehicle tracking problems. IEEE Transactions on Automatic Control, 59(10), 2801-2806.

Ali, H., Rudy R. N. (2019). Adaptive control for autonomous ships with uncertain model and unknown propeller dynamics. Control Engineering Practice, (19), 104116.

Arai, T., Pagello, E., \& Parker, L.E., (2002). Guest editorial advances in multi-robot systems. IEEE Transactions on Robotics \& Automation, 18(5), 655-661.

Bitar, G. , Lekkas, A. M. , \& Breivik, M. . (2019). Improvements to warm-started optimized trajectory planning for ASVs. https://arxiv.org/abs/1907.02696

Blaich, M., Rosenfelder, M., Schuster, M., Bittel, O., \& Reuter, J. . (2012). Fast Grid Based Collision Avoidance for Vessels using A * Search Algorithm. 17th International Conference on Methods and Models in Automation and Robotics (MMAR). IEEE.

Botea, A., Muller, M. and Schaeffer, J., 2004. Near optimal hierarchical path-finding. Journal of Game Development, (1):1-22

Caccia, M., Bibuli, M., Bono, R., \& Bruzzone, G.. (2008). Basic navigation, guidance 
and control of an Unmanned Surface Vehicle. Autonomous Robots, 25(4), 349365. doi: 10.1007/s10514-008-9100-0

Campbell, S., Naeem, W., (2012). A Rule-based Heuristic Method for COLREGscompliant Collision Avoidance for an Unmanned Surface Vehicle . IFAC Proceedings Volumes, 45(27):386-391.

Canny, J.F., (1998). The Complexity of Robot Motion Planning. MIT Press, Cambridge, MA.

Candeloro, M., Lekkas, A.M., Serensen, A.J., (2017) A Voronoi-diagram-based dynamic path-planning system for underactuated marine vessels. Control Engineering Practice, 61:41-54.

Casalino, G., Turetta, A., \& Simetti, E. . (2009). A three-layered architecture for real time path planning and obstacle avoidance for surveillance USVs operating in harbour fields. OCEANS 2009 - EUROPE. IEEE.

Chao, C. , Jian, T. , Zuguang, J. , Yang, Y. , \& Lei, Q., (2014). A path planning algorithm for seeing eye robots based on v-graph. Mechanical Science and Technology for Aerospace Engineering.

Chen C., Chen, X. Q., Ma, F., et al., (2019). A knowledge-free path planning approach for smart ships based on reinforcement learning. Ocean Engineering, 189, 1-9.

Chen, L., Huang, Y., Zheng, H., Hopman, H., \& Negenborn, R. (2019). Cooperative multi-vessel systems in urban waterway networks. IEEE Transactions on Intelligent Transportation Systems. DOI: 10.1109/TITS.2019.2925536

Cohen, I. , Epstein, C. , \& Shima, T. . (2017). On the discretized dubins traveling salesman problem. IISE Transactions, 49(2), 238-254.

Dahl, A.R., (2013). Path Planning and Guidance for Marine Surface Vessels. Institute for Teknisk Kybernetikk.

Dijkstra, E.W., (1959). A note on two problems in connection with graphs. Numerische Mathematik, 1, pp.269-271.

Djouani, K., \& Hamam, Y. (1995). Minimum time-energy trajectory planning for automatic ship berthing. IEEE Journal of Oceanic Engineering, 20(1), 4-12.

Durrant-Whyte Hugh. (1994). Where am I? A tutorial on mobile vehicle localization. Industrial Robot: An International Journal, 21(2), 11-16.

Escario, J.B., Jimenez, J.F., \& Giron-Sierra, J.M., (2012). Optimisation of autonomous ship manoeuvres applying ant colony optimisation metaheuristic. Expert Systems with Applications, 39(11), 10120-10139.

F. Li, "The Key Technology Research of Unmanned Surface Vehicle," M.S. thesis, dept. Electronics and Communication Engineering, Shandong Univ, Shandong, China, 2016.

F. Dougherty, \& G. Woolweaver, (1990). At-sea testing of an unmanned underwater 
vehicle flight control system. In Proceedings of the Symposium on Autonomous Underwater Vehicle Technology, pages 65-73. IEEE.

Fahey, S. , \& Luqi. (2016). Unmanned vehicles for anti-submarine warfare. Oceans. IEEE.

Farinelli, A., Iocchi, L., \& Nardi, D. (2004). Multi-robot systems: a classification focused on coordination. IEEE Transactions on Systems Man \& Cybernetics Part B Cybernetics A Publication of the IEEE Systems Man \& Cybernetics Society, 34(5), 2015-2028.

Fossen, T.I., (1994). Guidance and control of ocean vehicles . John Wiley \& Sons Inc.

Fossen, T.I., (2002). Marine control systems: guidance, navigation and control of ships, rigs and underwater vehicles . Marine cybernetics, Trondheim, Norway.

Fossen, T. I., Pettersen, K. Y., \& Nijmeijer, H., (2017). Sensing and Control for Autonomous Vehicles. Springer International Publishing.

G. Hitz et al., "State estimation for shore monitoring using an autonomous surface vessel”. Experimental Robotics, vol. 109, pp. 745-760, Nov 2016.

G. A. Wilde and R. R. Murphy, "User Interface for Unmanned Surface Vehicles Used to Rescue Drowning Victims," 2018 IEEE International Symposium on Safety, Security, and Rescue Robotics (SSRR), Philadelphia, PA, 2018, pp. 1-8.

G. Oriolo, M. Vendittelli, and G. Ulivi, "On-line map building and navigation for autonomous mobile robots," IEEE International Conference on Robotics \& Automation. IEEE, vol.3, June. 1995.

George, Y., Daniel, J.K., (2012). Ground Target Detection Using Cooperative Unmanned Aerial Systems. Journal of Intelligent \& Robotic Systems, (1).

Ghosh, S. K. and Mount, D. M. (1991). An output-sensitive algorithm for computing visibility graphs. SIAM Journal on Computing, 20(5), 888-910.

Gu, S., Zhou, C., Wen, Y., Xiao, C., Du, Z., Huang, L., (2019). Path Search of Unmanned Surface Vehicle Based on Topological Location . Navigation of China, 42(02):52-58.

Guangzhen Pa, Fan Qin, Wenbin Zhang. (2013). A Research for Track Planning Algorithm based on Dynamic Adaptive Rapidly-exploring Random Tree [J]. Microelectronics \& Compute, (1).

H. Choset, K.M. Lynch, S. Hutchinson, G.A. Kantor, W. Burgard, L.E. Kavraki, S. Thrun. (2005). Principles of Robot Motion: Theory, Algorithms, and Implementations, MIT Press.

Hao Yu. (2014). Nonholonomic wheeled mobile robot motion planning and control research. Ocean University of China.

Hart, P., Nilsson, N., and Raphael, B., (1968). A formal basis for the heuristic determination of minimum cost paths. IEEE Transactions on Systems Science and 
Cybernetics, 4(2), 100-107.

He, Y., Jin, Y., Huang, L., Xiong, Y. , Chen, P., \& Mou, J. . (2017). Quantitative analysis of COLREG rules and seamanship for autonomous collision avoidance at open sea. Ocean Engineering, 140, 281-291.

Heess, N., TB, D., Sriram, S., et al., (2017). Emergence of Locomotion Behaviours in Rich Environments. https://arxiv.org/abs/1707.02286v2.

Heoncheol, Lee., Touahmi, Yaniss., Beom-Hee, Lee., (2012). Grafting: A Path Replanning Technique for Rapidly-Exploring Trees in Dynamic Environments . Advanced Robotics, 26(18).

Hong Li, Wenjun Wang, Keqiang Li. (2016). Parallel parking path planning based on B-spline theory. China road journal, (09):143-151.

Hongwei, W. , Yong, M. A. , Yong, X. , \& Min, G. . (2010). Mobile robot optimal path planning based on smoothing A* algorithm. Journal of Tongji University (Natural Science), 38(11).

Huang Y., van Gelder, P.H.A.J.M., and Y. Wen. (2018). Velocity obstacle algorithms for collision prevention at sea. Ocean Engineering, 15, 308-321.

Huang Y., Linying Chen, P. H. A. J. M. van Gelder. (2018). Generalized Velocity Obstacle Algorithm for Preventing Ship Collisions at Sea. Ocean Engineering, DOI: 10.1016/j.oceaneng.2018.12.053

Huajun Liu, Jingyu Yang, Jianfeng Lu, Zhenming Tang, Chunxia Zhao, Weiming Cheng. (2006). A Review of Mobile Robot Motion Planning. Chinese Engineering Science, (01), 85-94.

Huai Yang. (2016). Research on path planning algorithm of USV. Daliann Maritime University.

Ihle, I. A. F. , Jouffroy, J., \& Fossen, T. I. . (2007). Formation control of marine surface craft: a lagrangian approach. IEEE Journal of Oceanic Engineering, 31(4), 922934.

J. Wang et al., "Design and Implementation of Ultra-long-endurance USV with Multiple Power Source," Ship Engineering, vol. 39, no. 5, pp.48-52+80, May. 2017.

J. T. Su, "Analysis of anti-submarine warfare module for unmanned surface vehicle," Ship Science and Technology, vol. 40, no. 2, pp. 135-139, Feb, 2018.

Jayoung Kim, Jihong Lee. (2013). A Testbed for Predicting Maneuverability of UGV on Rough Terrain. IFAC Proceedings Volumes, 46(10).

Jiayuan Zhuang, Lei Wan, Yulei Liao, Hanbing Sun. (2011). Global path planning of unmanned surface vehicle based on electronic chart. Computer Science, 38(09):211-214, 219.

Jiayuan Zhuang, Yuming Su, Yulei Liao, Hanbing Sun. (2012). Unmanned surface vehicle local path planning based on marine radar. Journal of Shanghai Jiaotong 
University, 46(09):1371-1375, 1381.

Jian, L. (2014). Research on path planning technique of unmanned surface vehicle. Jiangsu University of Science and Technology.

Jinze Song, Bin Dai, Enzhong Shan. (2010). An improved RRT path planning algorithm . Acta Electronica Sinica, 38:225-228.

Jing, L., Jiang, L., \& Hongli, X. U.. (2015). Online path planning of an unmanned surface vehicle for real-time collision avoidance. CAAI Transactions on Intelligent Systems.

Jin, K. F., Wang, H. D., Yi, H., et al., (2018). Key technologies and intelligence evolution of maritime UV. Chinese Journal of Ship Research, 13(6), 1-8.

KavrakiL, LatombeJ. (1994) Randomized preprocessing of configuration space for fast path planning. IEEE int confon Robotics and Automation. SanDiego, 2138 2139.

Kim, H., Kim, D., Shin, J. U., Kim, H., \& Myung, H. (2014). Angular rate-constrained path planning algorithm for unmanned surface vehicles . Ocean Engineering, 84(4), $37-44$.

Kim, J. , Kim, M. , \& Kim, D. . (2011). Variants of the quantized visibility graph for efficient path planning. Advanced Robotics, 25(18), 2341-2360.

Kohei Shiozawa. (2016). Revealed preference test and shortest path problem; graph theoretic structure of the rationalizability test. Journal of Mathematical Economics, 67:38-48.

Koenig, S., Likhachev, M., \& Furcy, D. (2004). Lifelong planning A*. Artificial Intelligence, 155(1-2), 93-146.

Kui Yuan, Yuan Li, Lixin Fang. (2007). Recent researches and development on multirobot system . Journal of automation, 33(8), 785-794.

Latip, N. B. A., Omar, R., \& Debnath, S. K. . (2017). Optimal path planning using equilateral spaces oriented visibility graph method. International Journal of Electrical \& Computer Engineering, 7(6), 3046-3051.

Lazarowska, A. (2015). Ship's trajectory planning for collision avoidance at sea based on ant colony optimisation. Journal of Navigation, 68(02), 291-307.

Lazarowska, A. (2020). A Discrete Artificial Potential Field for Ship Trajectory Planning. Journal of Navigation, 73(1), 233-251.

Lei X. et al., (2019). A path planning approach based on multi-direction A* algorithm for ships navigating within wind farm waters. Ocean engineering, 184, 311-322.

Lekkas A M. (2014). Guidance and Path-Planning Systems for Autonomous Vehicles. Norwegian University of Science and Technology, Department of Engineering Cybernetics.

Li, S., Liu, J., \& Negenborn, R. R. (2019). Distributed coordination for collision avoidance of multiple ships considering ship maneuverability. Ocean Engineering, 
$181,212-226$.

Liang Hu., Wasif Naeem., Eshan Rajabally., Graham Watson., Terry Mills., Zakirul Bhuiyan. (2019) A multi-objective optimization approach for colregs-compliant path planning of autonomous surface vehicles verified on networked bridge simulators. IEEE Transactions on Intelligent Transportation Systems, PP(99), 113.

Liang Kang. (2010). Several algorithmic researches on motion planning for autonomous mobile robot . Nanjing University of Science and Technology.

Lillicrap, T. P., Hunt, J. J. , Pritzel, A., Heess, N. , Erez, T. , \& Tassa, Y., et al. (2015). Continuous control with deep reinforcement learning. Computer Science, 8(6), A187.

Liu, B., Chen, Z., Zhang, H. T., Wang, X., Geng, T., Su, H., \& Zhao, J. (2019). Collective Dynamics and Control for Multiple Unmanned Surface Vessels. arXiv preprint arXiv:1905.01215.

Liu, Z., Zhang, Y., Yu, X., Yuan, C. (2016). Unmanned surface vehicles: An overview of developments and challenges. Annual Reviews in Control, 41, 71-93.

Liu, S., Sun, D., \& Zhu, C. . (2011). Coordinated motion planning for multiple mobile robots along designed paths with formation requirement. IEEE/ASME Transactions on Mechatronics, 16(6), 1021-1031.

Liu L., Xiao C., Wen Y.. (2017). Motion planning algorithm for unmanned surface vehicle based on Dubins path. Journal of Computer Applications, 37(07):2114$2117+2123$.

Liu, Y. , \& Bucknall, R. . (2016). The angle guidance path planning algorithms for unmanned surface vehicle formations by using the fast marching method. Applied Ocean Research, 59, 327-344.

Lydia E. Kavraki and Steven M. LaValle. (2008). Motion planning . Springer Handbook of Robotics, 109-131.

M. Pivtoraiko and A. Kelly. (2005) "Generating near minimal spanning control sets for constrained motion planning in discrete state spaces," in Proceedings of the IEEE International Conference on Intelligent Robots and Systems (IROS).

Maulik, U., \& Bandyopadhyay, S. (2000). Genetic algorithm-based clustering technique. Pattern Recognition, 33(9), 1455-1465.

Ma, Y., Hu, M., \& Yan, X. (2018). Multi-objective path planning for unmanned surface vehicle with currents effects. ISA transactions, 75, 137-156.

Martinsen, A. B., Lekkas, A. M., \& Gros, S. (2019). Autonomous docking using direct optimal control. arXiv preprint arXiv:1910.11625.

Meyer, P. J., Yin, H., Brodtkorb, A. H., Arcak, M., \& Sørensen, A. J. (2019). Continuous and discrete abstractions for planning, applied to ship docking. arXiv preprint arXiv:1911.09773. 
Mingzhu Wang. (2016). The Application Status and Prospect of Civil UAV. Proceedings of the 2016 China International Unmanned Aircraft Systems Conference. China Aviation Society, May.

Mingbo Du, Tao Mei, Jiajia Chen et al. (2015). RRT-based Motion Planning Algorithm for Intelligent Vehicle in Complex Environments. ROBOT, 04:443-450.

Michael RG, Johnson DS. (1979). Computers and intractability: a guide to the theory of np-completeness. WH Free Co, San Fr 1979:90-1.

Murphy, R.. (2000). Introduction to AI robotics. Massachusetts: MIT Press.

Naeem, W., Henrique, S. C., Hu, L. (2016). A Reactive COLREGs-Compliant Navigation Strategy for Autonomous Maritime Navigation. IFAC-PapersOnLine, 49(23), 207-213.

$\mathrm{Na} \mathrm{Xu}$, Xiong Chen, Qingsheng Kong, Jianda Han. (2011). Motion planning for robot with nonholonomic constraints. ROBOT, 06:666-672.

Nan Feng. (2014). A path planning method for autonomous mobile robot based on RRT algorithm. Dalian University of Technology.

Niu, H., Savvaris, A., Tsourdos, A., \& Ji, Z. (2019). Voronoi-Visibility Roadmap-based Path Planning Algorithm for Unmanned Surface Vehicles. Journal of Navigation, 72(4), 850-874. doi:10.1017/S0373463318001005

Niu, H., Lu, Y., Savvaris, A., \& Tsourdos, A. (2016). Efficient Path Planning Algorithms for Unmanned Surface Vehicle. 10th IFAC Conference on Control Applications in Marine Systems (CAMS 2016). IEEE.

Okazaki, T., Ohtsu, K., \& Mizuno, N. (2000). A study of minimum time berthing solutions. IFAC Proceedings Volumes, 33(21), 135-139.

Ogawa, O., 1977. MMG Report I: Mathematical Model of Control Movement. The Japan Shipbuilding Society.

P. Svec, A. Thakur, B. C. Shah, and S. K. Gupta. (2012). USV Trajectory Planning for Time Varying Motion Goals in an Environment with Obstacles. ASME 2012 International Design Engineering Technical Conferences (IDETC) \& Computers and Information in Engineering Conference (CIE), August 12-15, Chicago, USA.

Patron, P. (2010). Semantic-based adaptive mission planning for unmanned underwater vehicles (Doctoral dissertation, Heriot-Watt University).

Plaku, E., Bekris, K.E., Chen, B.Y., Ladd, A.M., Kavraki, L.E., (2005). Sampling based roadmap of trees for parallel motion planning, IEEE Transactions on Robotics 21 (4), 597-608.

Qu, H. , Xing, K. , \& Alexander, T. . (2013). An improved genetic algorithm with coevolutionary strategy for global path planning of multiple mobile robots. Neurocomputing, 120(10), 509-517.

Rong, H., Teixeira, A. P., Guedes, Soares, C.. (2019).Ship trajectory uncertainty 
prediction based on a Gaussian Process model. Ocean Engineering, 182, 499-511.

S. M. LaValle. (1998). Rapidly-exploring Random Trees: a new tool for path planning, Technical Report TR98-11, Computer Science Dept, Iowa State University.

S. Zhao et al., "USV Based on Automatic Stabilizing Function," Ordnance Industry Automation, vol. 37, no. 3, pp. 32-35, Mar. 2018.

Sakamoto, N. (2013). Case studies on the application of the stable manifold approach for nonlinear optimal control design. Automatica, 49(2), 568-576.

Sarda, E. I., Qu, H., Bertaska, I. R., \& Von Ellenrieder, K. D., (2016). Station-keeping control of an unmanned surface vehicle exposed to current and wind disturbances. Ocean Engineering, 127, 305-324.

Simetti, E. Turetta, A., Casalino, G., Storti, E., \& Cresta, M. (2009). Towards the use of a team of USVs for civilian harbour protection: Real time path planning with avoidance of multiple moving obstacles. In IEEE 3rd workshop on planning, perception and navigation for intelligent vehicles (IROS'09), St. Louis.

Singh, Y. et al., (2018). A constrained A* approach towards optimal path planning for an unmanned surface vehicle in a maritime environment containing dynamic obstacles and ocean currents. Ocean Engineering, 169:187-201

Shafer, A. J., Benjamin, M. R., Leonard, J. J., \& Curcio, J. (2008). Autonomous cooperation of heterogeneous platforms for sea-based search tasks. In Proceedings of MTS/IEEE OCEANS (pp. 1-10).

Shi, Y., \& Eberhart, R. C. (2002). Empirical study of particle swarm optimization. Congress on Evolutionary Computation.

Shijie, L. Jialun, L. Rudy, R.N. (2019). Distributed coordination for collision avoidance of multiple ships considering ship maneuverability. Ocean Engineering, 181, 212226.

Shi, B., Su, Y., Wang, C., Wan, L., L, Y., (2019). Study on intelligent collision avoidance and recovery path planning system for the waterjet-propelled unmanned surface vehicle. Ocean Engineering, 182, 489-498.

Song, R., Liu, Y. , \& Bucknall, R. . (2017). A multi-layered fast marching method for unmanned surface vehicle path planning in a time-variant maritime environment . Ocean Engineering, 129, 301-317.

Song, R., Liu Y., Bucknall R . (2019). Smoothed A* algorithm for practical unmanned surface vehicle path planning. Applied ocean research, 83:9-20.

Steimle, E. T. \& Hall, M. L. (2006). Unmanned surface vehicles as environmental monitoring and assessment tools. In OCEANS (pp. 1-5).

Steimle, E., Murphy, R., Lindemuth, M. and Hall, M., (2009). Unmanned marine vehicle use at hurricanes wilma and ike. In Proceedings of MTS/IEEE Oceans'09, Biloxi, MS, U.S.A., 6 - 29 October 2009, pp.1-6

Steven M. LaValle. (2006). Planning Algorithms . Cambridge University Press, Cambridge, UK. 
Svec, P. , Schwartz, M. , Thakur, A. , \& Gupta, S. K. . (2011). Trajectory Planning with Look-Ahead for Unmanned Sea Surface Vehicles to Handle Environmental Disturbances. 2011 IEEE/RSJ International Conference on Intelligent Robots and Systems, IROS 2011, San Francisco, CA, USA, September 25-30, 2011. IEEE.

T. H. Yang et al., "Development of unmanned surface vehicle for water quality monitoring and measurement," 2018 IEEE International Conference on Applied System Invention (ICASI), Chiba, 2018, pp. 566-569.

Thakur, A., Svec, P., \& Gupta, S. K. . (2011). Generation of state transition models using simulations for unmanned sea surface vehicle trajectory planning. Robotics \& Autonomous Systems, 60(12), 1457-1471.

U.S. Department of Homeland Security, U.S. Coast Guard, (2010). Navigation Rules. Paradise Cay Publications.

Vettor, R. , \& Guedes Soares, C. . (2016). Development of a ship weather routing system. Ocean Engineering, 123, 1-14.

Vitor A. M. Jorge, Roger Granada, Renan G. (2019). Maidana, et al. A Survey on Unmanned Surface Vehicles for Disaster Robotics: Main Challenges and Directions. Sensors, 19, 702.

W. Naeem et al., "The design of a navigation, guidance, and control system for an unmanned surface vehicle for environmental monitoring," Proceedings of the Institution of Mechanical Engineers, Part M: Journal of Engineering for the Maritime Environment, vol. 222, no. 2, pp. 67-79, May, 2008.

W. Z. Li, J. P. Song and L. Y. Liu, "Analysis of the military requirement and operational mode of the ACTUV," Defense Technology Review, vol. 39, no. 4, pp.69-71, Apr, 2018.

W. J. Zeng, "Marine objection detection and tracking of USV based on visual system," $\mathrm{Ph} . \mathrm{D}$. dissertation, dept. Design and manufacture of ships and marine structures, Harbin Engineering University, Harbin, China, 2013.

Wang, Chengbo, Zhang, Xinyu, Li, Ruijie, \& Dong, Peifang. (2019). Path Planning of Maritime Autonomous Surface Ships in Unknown Environment with Reinforcement Learning, Singapore.

Wang, C., Cheng, J., Chi, W., Yan, T., \& Meng, M. Q. H. (2019). Semantic-Aware Informative Path Planning for Efficient Object Search Using Mobile Robot. IEEE Transactions on Systems, Man, and Cybernetics: Systems.

Watkins, C.J.C.H., Dayan, P., 1992. Q-learning. Mach. Learn. 8, 279-292.

Wang, N., Jin, X., Joo, Er, M.. (2019). A multilayer path planner for a USV under complex marine environments. Ocean Engineering, 184,1-10.

Wen, Y., Zhang, Y., Huang, L., Zhou, C., Xiao, C., Zhang, F., ... \& Sui, Z. (2019). Semantic Modelling of Ship Behavior in Harbor Based on Ontology and Dynamic Bayesian Network. ISPRS International Journal of Geo-Information, 8(3), 107.

X. J. Chen et al., "Unmanned boat target detection and tracking method based on SSDCF," China measurement and test, vol. 45, no. 2, pp. 145-149, Feb.2019.

X. Y. Cong, J. H. Wang and Z. Y. Li, "Research of Target Path Tracking Optimization Control of Unmanned Surface Vehicle," Computer Simulation, vol. 36, no. 1, pp. 
325-329, Jan, 2019.

X. D. Tan, "Research on the Speed and Course Control of SRUSV. Master's thesis, dept. Mechanical engineering, Zhejiang University, Zhejiang, China, 2019.

Xiaojie Sun. (2016). Research on the real-time path planning system of unmanned surface vehicle. Daliann Maritime University.

Xu H, Guedes Soares, C. (2016). Vector field path following for surface marine vessel and parameter identification based on LS-SVM. Ocean Engineering, 2113:151161.

Y. Peng et al., "Sea surface object tracking for USV with spatio-temporal context fusion," Scientia Sinica Technologica, vol. 48, no.12, pp. 103-118, Nov.2018.

Yaagoubi, R., Miky, Y., \& El Shouny, A. (2020) AccessVOR: A Semantically Enriched Voronoï-Based Approach for Navigation Assistance of Wheelchair Users in Indoor Environments. The Journal of Navigation, 73(1), 172-191.

Yanshuang Lu. (2010). Research on path planning algorithm of unmanned surface vehicle. Harbin Engineering University.

Yang, J. M., Tseng, C. M., \& Tseng, P. S. (2015). Path planning on satellite images for unmanned surface vehicles. International Journal of Naval Architecture \& Ocean Engineering, 7(1), 87-99.

Yang, S. X., Luo, C. . (2004). A neural network approach to complete coverage path planning. IEEE Transactions on Systems Man \& Cybernetics Part B Cybernetics A, 34(1), 718-725.

Yi Chen. (2016). A preliminary study on the optimized collaborative strategy and implementation of the unmanned surface vehicle. Jiangsu University of Science and Technology.

Yi Wang, Xiaopin Zhu, Zhou Zhou. (2012). A method of UAV path planning based on the Clothoid curve. Journal of Northwestern Polytechnical University, (06):874878.

Yoo, B. , \& Kim, J. . (2016). Path optimization for marine vehicles in ocean currents using reinforcement learning. Journal of Marine Science and Technology, 21(2), 334-343.

Yuxuan, Z. , Lei, G. E. , Xin, Z. , Yan, P. , Yi, Y. , \& Xiaomao, L. I. . (2017). Complete coverage path planning of USV used for mapping round island. Journal of Shanghai University(Natural Science Edition).

Zhang, F., Marani, G., Smith, R. N., \& Choi, H. T., (2015). Future trends in marine robotics [tc spotlight]. IEEE Robotics \& Automation Magazine 22(1), 14-122.

Zhang, Y., Wen, Y., Zhang, F., Zhou, C., Du, L., Huang, L., \& Xiao, C. (2018). Semantic model of ship behaviour based on ontology engineering. The Journal of Engineering, 2018(16), 1746-1752. 
Zhe Du et al., (2018). Motion planning for Unmanned Surface Vehicle based on trajectory unit . Ocean Engineering, vol. 151, no. 1, pp. 46-56, Mar. 2018.

Zhe Du et al., (2019). Trajectory-cell based method for the unmanned surface vehicle motion planning. Applied Ocean Research, Vol. 86, pp. 207-221, May 2019.

Zheng, J., \& Zhu, G. (2019). A weak maximum principle-based approach for input-tostate stability analysis of nonlinear parabolic PDEs with boundary disturbances. arXiv preprint arXiv:1908.00335.

Zhou, Z., (2016). Machine Leaning . Beijing: Tsinghua University Press.

Zhuang, J. Y., Su, Y. M. , Liao, Y. L. , \& Sun, H. B. . (2011). Motion planning of USV based on marine rules. Procedia Engineering, 15, 269-276.

Zuquan Xiang, Chao Jin, Kaijun Du, Yunsheng Mao, Lifei Song. (2015). Local obstacle avoidance for unmanned surface vehicle using a hierarchical strategy based on particle swarm optimization [J]. Journal of wuhan university of technology, 37(07):38-45. 